\title{
Effect of micronutrients foliar application and biofertilizeres on essential oils of lemon balm
}

\author{
M. Yadegari*
}

Department of Agronomy and Medicinal Plants, Faculty of Agriculture, Shahrekord Branch, Islamic Azad University, Po. Box:166. Shahrekord, Iran.*Correspondingauthor: mehrabyadegari@gmail.com

\begin{abstract}
In this work, the effects of two micronutrients $\left(\mathrm{Mn}^{2+}, \mathrm{H}_{2} \mathrm{Bo}^{3-}\right)$ and macronutrients $\left(\mathrm{PO}_{4}^{3-}\right.$ and $\left.\mathrm{NO}_{3}^{-}\right)$from biofertilizeres resources on growth (leaf dry/fresh weight, root dry/fresh weight, stem/root length, number of stem) and accumulation of essential oils in Lemon balm (Melissa officinalis L.) were investigated. The most of essential oils in two seasons were Caryophyllene oxide, E-Caryophyllene, Geranial, Geraniol, Chavicol and Neral that affected by the treatments. Of the two micronutrients, manganese, and in macronutrients $\mathrm{NO}_{3}^{-}$were more effective in stimulating the accumulation of components. At $150 \mathrm{ppm}$ of micronutrients by biofertilizeres, enhanced the production of citronellal, Chavicol. Although combination of $\mathrm{H}_{2} \mathrm{Bo}^{3-}$ and $\mathrm{Mn}^{2+}$ at $300 \mathrm{ppm}$ by macronutrients in some of essential oils like neral, caryophyllene oxide and 14-hydroxy-Z-caryophyllene were more produced than $150 \mathrm{ppm}$ combinations but most of essential oils significantly increased in $150 \mathrm{ppm}$ concentration of micronutrients with macronutrients. Exo-Isocitral, Chavicol, 14-hydroxy-Z-Caryophyllene and Germacrene $\mathrm{D}$, were upper extracted in $150 \mathrm{ppm}$ concentration of micronutrients but in many combinations this essential oil was no extracted in little concentration.
\end{abstract}

Keywords: Lemon balm, nutrients, phytochemical 


\section{Introduction}

Lemon balm (Melissa officinalis L.) from Lamiaceae family, a native of the northern Mediterranean region is cultivated as a medical herb. It is listed in a number of European Pharmacopoeia for its carminative, digestive, diaphoretic and stimulant activities (Sekeroglu et al. 2006). Lemon balm is one of the most important medicinal and aromatic plants, with antioxidant, antimicrobial, spasmolytic, astringent and specific sensorial properties. Essential oil of the plant, composed mainly of contains caryophyllene $\beta$, citronellal, geraniol, geranyl acetate, linalool, neral and ursolic acid, is responsible for some of these effects (Bagdat and Cosge, 2006). Small amount of $\mathrm{Mn}^{2+}$ and $\mathrm{H}_{2} \mathrm{Bo}^{3-}$ are essential for growth and quality of the crop because these micronutrients also control most of the physiological activities of the crop by interrupting the level of chlorophyll content in leaves which ultimately influence the photosynthetic activity of the plant. In micronutrients, Manganese is involved in many biochemical functions, primarily acting as an activator of enzymes such as dehydrogenases and decarboxylases involved in respiration, amino acid and lignin synthesis, and hormone concentrations (Galavi et al. 2012). Boron made resistance of plasmalema and combination by other minerals and necessary for plants (Widom and Mihalkovic, 2008). Foliar fertilizer is particularly useful technique which can be designed to meet plants specific needs for one or more micro or macronutrients especially trace minerals and enable to correct deficiencies, strengthen weak or damaged crops, speed growth and grow better and healthier plants. In a sense, biofertilizeres (beneficial microbial populations) are unique alternatives for the sustainable agriculture and safe production. Microbial inoculums not only increased the nutritional assimilation of plant (total N, P and K), but also improved soil properties, such as organic weight content, dissolved organic carbon, dissolved organic nitrogen and total $\mathrm{N}$ in soil (Ge et al. 2014; Adak et al. 2014). Biofertilizeres have the social community of bacteria and other microorganisms that live in rhizosphere, colonize upon roots and as a consequence of this symbiotic relationship; the host plant achieves a better growth. For instance, plant growth-promoting rhizobacteria (PGPR) are beneficial native soil bacteria that colonize on plant roots and result in increased plant growth (Yadegari et al. 2010). Micro and macro elements play an important role in promoting the growth and production of plants. Micro elements participate in most of the enzymatic reaction and they also play a role indirectly through the synthesis of several growth regulators (Yadegari, 2015). N addition increased the soil microbial biomass carbon (SMBC) and soil bacterial diversity. Moderate $\mathrm{N}$ $\left(23,46,69 \mathrm{~kg} \mathrm{ha}^{-1} \mathrm{yr}^{-1}\right)$ addition increased the soil bacterial diversity, whereas excess $\mathrm{N}\left(92 \mathrm{~kg} \mathrm{ha}^{-1} \mathrm{yr}^{-1}\right)$ addition inhibited it. The SMBC and soil bacterial diversity were related to richness of plant functional groups (Sun et al. 2014). Fertilisation with micronutrients can be effective in increasing the concentration of micronutrient sat the soil-root interface. In addition, micronutrient-efficient crops and genotypes can increase an available nutrient fraction and hence increase micronutrient uptake. Physiological processes governing exudation and the soil-plantmicrobe interactions in the rhizosphere is currently inadequate, especially in terms of spatial and temporal variability in root exudation as well as the fate and effectiveness of organic and inorganic compounds in increasing availability of soil micronutrients and undesirable trace elements. The interactions between microorganisms and plants at the soil root interface are particularly important as well obscure (Rengl, 2015; Viruel et al. 2014). 
Although many studies performed about of macronutrients effects on plants (Quezada et al. 2007; Wyllyam Do Vale et al. 2008; Khan et al. 2014; Raza et al. 2014; Sandana and Pinochet, 2014; Yañez-Mansilla et al. 2015), few studies examining micronutrients fertilizers and interaction of them by macronutrients in essential oil components on lemon balm have been conducted. Objectives of this study were to determine the effects of Manganese and Boron foliar applications with macronutrients $\left(\mathrm{PO}_{4}^{3-}\right.$ and $\left.\mathrm{NO}_{3}^{-}\right)$from biofertilizeres resources on some of plant growth parameters (leaf dry/fresh weight, root dry/fresh weight, stem/root length, number of stem) and essence components such as Neral, Chavicol, Geraniol, Geranial, E-Caryophyllene, Caryophyllene oxide of Melissa officinalis L. at two seasons.

\section{Materials and Methods}

Seeds of Lemon balm were obtained from Iranian Seeds and Plant Improvement Institute. Seeds were planted in field condition. Table 1 shows physicochemical properties of the soil. Treatments were micronutrient $\mathrm{Mn}$ and $\mathrm{B}$ levels and macronutrients $\left(\mathrm{PO}_{4}^{3-}\right.$ and $\left.\mathrm{NO}_{3}^{-}\right)$from biofertilizeres resources. Field trials were established in 2013 and 2014 at Shahrekord (50 56/ E 32 $18 /$ N) South Western Iran. Experiments were arranged in a randomized complete block design with a factorial layout and three replications. Topsoil of the experimental plot area was kept moist throughout the growing season when necessary.

Table 1. Some physical and chemical properties of soil for experiment $(0-30) \mathrm{cm}$.

\begin{tabular}{|c|c|c|c|c|c|c|c|c|c|c|c|}
\hline \multirow{2}{*}{ Year } & \multirow{2}{*}{ Texture } & \multirow{2}{*}{$\begin{array}{c}\text { E.C } \\
\left(\text { ds. }^{-1}\right)\end{array}$} & $\mathrm{N}_{\text {total }}$ & O.C & \multirow{2}{*}{$\mathrm{pH}$} & K & $\mathrm{P}$ & $\mathrm{Zn}$ & $\mathrm{Mn}$ & $\mathrm{Fe}$ & $\mathrm{Cu}$ \\
\hline & & & 0 & & & \multicolumn{6}{|c|}{$\mathrm{mg} \cdot \mathrm{kg}^{-1}$} \\
\hline 1 & & 8.1 & 0.11 & 0.2 & & 770 & 45 & 0.57 & 1.2 & 4.1 & 1.3 \\
\hline 7 & Loam & 7.8 & 0.11 & 0.2 & 8.5 & 745 & 44 & 0.45 & 1.1 & 3.1 & 1.1 \\
\hline
\end{tabular}

Two foliar fertilizers Librel Mn and Unibor were applied and all of them are mineral fertilizers. Librel $\mathrm{Mn}$ inclusive of $13 \% \mathrm{Mn}$ chelated with EDTA and Unibor contains 15\% B (obtained from The Chemical Company of England and Germany). Theses fertilizers were sprayed at three concentrations $\left(\mathrm{Mn}_{1}, \mathrm{Mn}_{2}\right.$, $\mathrm{Mn}_{3}$ were 0,150 and $300 \mathrm{ppm}$ of $\mathrm{Mn}$, respectively and similar in B).

Phosphate-solubilizing bacterium, Bacillus licheniformis, in powder form applied at the level of $100 \mathrm{gr}^{- \text {ha }^{-1}}$ $\left(\mathrm{P}_{1}\right.$ and $\mathrm{P}_{2}$ were used as symbols to show soil fortified with biofertilizer and without respectively). Nitroxin bio-fertilizer which its effective site is the group of nitrogen fixing bacteria belongs to Azospirillum and Azotobacter genera in liquid form applied at the level of 4 1.ha ${ }^{-1}\left(\mathrm{~N}_{1}\right.$ and $\mathrm{N}_{2}$ were used as symbols to show soil fortified with biofertilizer and without respectively). After soil test, the required nutrients were added to soil. At the beginning of the blooming stage, shoots of plants were harvested. Lemon balm shoots were cleaned with distillated water, and then were dried 3 days at $40{ }^{\circ} \mathrm{C}$ in oven. Micronutrients concentrations $\left(\mathrm{Mn}^{2+}\right.$ and $\mathrm{H}_{2} \mathrm{Bo}^{3-}$ in 0,150 and $300 \mathrm{ppm}$ ) were employed since of planting to beginning of flowering of plants in two steps by 2 week distance. The essential oils were analyzed by gas chromatography-mass spectrometry (GC/MS). Thermo Finnegan Trace 2000 GC/MS, made in the USA, was employed with a HP-5MS capillary column (30 m long and $0.25 \mathrm{~mm}$ wide, and a $0.25 \mu \mathrm{m}$ of film thickness) at a $250{ }^{\circ} \mathrm{C}$ of injector chamber. 
The initial column temperature was at $120^{\circ} \mathrm{C}$ for 5 min then raised to $280{ }^{\circ} \mathrm{C}$ at the rate of $10{ }^{\circ} \mathrm{C} / \mathrm{min}$. Helium was used as a carrier gas at a rate of $35 \mathrm{ml} /$ min. MS parameters were as follows: ionization energy, $70 \mathrm{eV}$; ion source temperature, $200^{\circ} \mathrm{C}$; voltage, $3000 \mathrm{v}$; and mass range, 30 to 600 . The compositions of the essential oil were identified by comparison of their retention indexes, retention times and mass spectra with those of authentic samples in Wiley library (Adams, 2001).

Amounts of essential oils components production as well as the chemical component of the essence were determined.

All data were subjected to ANOVA using the statistical computer package SAS ver.8 and treatment means separated using L.S.D multiple range test at $\mathrm{P}<0.05$ level.

\section{Results}

In various components, the best results made by consumption of 150 and $300 \mathrm{ppm}$ of micronutrients with use of biofertilizeres. The most of essential oils in two seasons were Caryophyllene oxide, E-Caryophyllene, Geranial, Geraniol, Chavicol and Neral that affected by the treatments (Tables 3-6). Also, the micronutrients application affected the caryophyllene oxide, E-Caryophyllene, Geranial, Geraniol, Chavicol and Neralpercentage. Similarly, the application of biofertilizeres increased the Caryophyllene oxide, E-Caryophyllene, Geranial, Geraniol and Chavicol of percentage by $22 \%$. The foliar application of $\mathrm{H}_{2} \mathrm{Bo}^{3-}$ and $\mathrm{Mn}^{2+}$ affected the Caryophyllene oxide, E-Caryophyllene, Geranial, Geraniol, Chavicol and Neralof concentration an average of $34 \%$ at $150 \mathrm{ppm}$ application of micronutrients compared with the control (Tables 3-4).

Table 2a. Complex analysis of variance of essential oils components in essence in Lemon balm plants that are affected by micronutrients and biofertilizeres.

\begin{tabular}{|c|c|c|c|c|c|c|c|c|}
\hline \multirow[t]{2}{*}{ Source of variation } & \multirow[t]{2}{*}{$\begin{array}{l}\text { Degree of } \\
\text { freedom }\end{array}$} & $\begin{array}{l}\text { Leaf dry } \\
\text { weight }\end{array}$ & $\begin{array}{c}\text { Leaf Fresh } \\
\text { weight }\end{array}$ & $\begin{array}{l}\text { Root dry } \\
\text { weight }\end{array}$ & $\begin{array}{c}\text { Root } \\
\text { Fresh } \\
\text { weight }\end{array}$ & $\begin{array}{l}\text { Stem } \\
\text { length }\end{array}$ & $\begin{array}{l}\text { Root } \\
\text { length }\end{array}$ & $\begin{array}{c}\text { Number of } \\
\text { stem }\end{array}$ \\
\hline & & \multicolumn{7}{|c|}{ Mean of Square } \\
\hline Year(Y) & 1 & $5.5^{*}$ & $10.1^{*}$ & $4.7^{*}$ & $7.5^{\mathrm{ns}}$ & $24.1^{\mathrm{ns}}$ & $21.6^{*}$ & $28.9^{*}$ \\
\hline $\mathrm{R} / \mathrm{Y}$ & 4 & $3.4^{*}$ & $6.22^{*}$ & $2.91^{*}$ & $5.1^{*}$ & $15.1^{*}$ & $13.36^{*}$ & $17.98^{*}$ \\
\hline Manganese(Mn) & 2 & $7.5^{* *}$ & $12.6^{* *}$ & $3.67^{*}$ & $6.4^{*}$ & $18.9^{*}$ & $16.8^{*}$ & $22.65^{*}$ \\
\hline Boron (B) & 2 & $7.7^{* *}$ & $13.1^{* *}$ & $3.6^{*}$ & $6.3^{*}$ & $19.1^{*}$ & $16.9^{*}$ & $22.71^{*}$ \\
\hline Nitrogen (N) & 1 & $6.8^{* *}$ & $15.91^{* *}$ & $8.4^{* *}$ & $8.2^{*}$ & $24.4^{*}$ & $21.7^{*}$ & $29.1^{*}$ \\
\hline Phosphorus (P) & 1 & $7.8^{* *}$ & $22.62^{* *}$ & $10.1^{* *}$ & $8.3^{*}$ & $24.2^{*}$ & $21.9^{*}$ & $29.2^{*}$ \\
\hline $\mathrm{Mn} \times \mathrm{B}$ & 4 & $4.7^{* *}$ & $12.1^{* *}$ & $5.5^{* *}$ & $5.12^{*}$ & $15.2^{*}$ & $13.35^{*}$ & $18.1^{*}$ \\
\hline $\mathrm{Mn} \times \mathrm{N}$ & 2 & $5.7^{* *}$ & $13.2^{* *}$ & $6.6^{* *}$ & $8.8^{* *}$ & $41.1^{* *}$ & $33.1^{* *}$ & $41.1^{* *}$ \\
\hline $\mathrm{Mn} \times \mathrm{P}$ & 2 & $6.9^{* *}$ & $12.2^{* *}$ & $7.76^{* *}$ & $9.3^{* *}$ & $39.5^{* *}$ & $33.7^{* *}$ & $43.2^{* *}$ \\
\hline $\mathrm{B} \times \mathrm{N}$ & 2 & $6.2^{* *}$ & $17.2^{* *}$ & $8.91^{* *}$ & $11.1^{* *}$ & $51.6^{* *}$ & $42.4^{* *}$ & $51.5^{* *}$ \\
\hline $\mathrm{B} \times \mathrm{P}$ & 2 & $5.9^{* *}$ & $13.4^{* *}$ & $9.78^{* *}$ & $12.4^{* *}$ & $52.4^{* *}$ & $45.5^{* *}$ & $56.7^{* *}$ \\
\hline $\mathrm{N} \times \mathrm{P}$ & 1 & $5.9^{* *}$ & $32.43^{* *}$ & $12.3^{* *}$ & $11.2^{* *}$ & $45.5^{* *}$ & $40.2^{* *}$ & $29.05^{*}$ \\
\hline $\mathrm{Mn} \times \mathrm{B} \times \mathrm{N}$ & 4 & $4.8^{*}$ & $14.5^{* *}$ & $6.8^{* *}$ & $8.5^{* *}$ & $28.8^{* *}$ & $28.1^{* *}$ & $18.2^{*}$ \\
\hline $\mathrm{Mn} \times \mathrm{B} \times \mathrm{P}$ & 4 & $4.8^{*}$ & $14.1^{*}$ & $8.81^{* *}$ & $9.5^{* *}$ & $44.2^{* *}$ & $29.9^{* *}$ & $18.3^{*}$ \\
\hline $\mathrm{Mn} \times \mathrm{N} \times \mathrm{P}$ & 2 & $4.3^{*}$ & $7.8^{*}$ & $10.1^{* *}$ & $8.1^{* *}$ & $61.1^{* *}$ & $41.7^{* *}$ & $54.2^{* *}$ \\
\hline $\mathrm{B} \times \mathrm{N} \times \mathrm{P}$ & 2 & $4.12^{*}$ & $12.6^{* *}$ & $12.2^{* *}$ & $9.6^{* *}$ & $59.2^{* *}$ & $45.8^{* *}$ & $56.1^{* *}$ \\
\hline $\mathrm{Mn} \times \mathrm{B} \times \mathrm{N} \times \mathrm{P}$ & 4 & $3.4^{*}$ & $16.2^{* *}$ & $9.8^{* *}$ & $4.95^{*}$ & $42.5^{* *}$ & $32.5^{* *}$ & $34.5^{* *}$ \\
\hline $\mathrm{T}(\mathrm{Mn} \times \mathrm{B} \times \mathrm{N} \times \mathrm{P}) \times \mathrm{Y}$ & 35 & $2.1^{*}$ & $3.84^{*}$ & $1.8^{*}$ & $3.14^{*}$ & $9.3^{*}$ & $8.3^{*}$ & $11.1^{*}$ \\
\hline $\mathrm{E}$ & 140 & 1.4 & 2.56 & 1.2 & 2.1 & 6.2 & 5.5 & 7.4 \\
\hline \multicolumn{2}{|c|}{ Coefficient of variation } & 2.74 & 5.5 & 1.5 & 3.4 & 8.9 & 5.9 & 5.3 \\
\hline
\end{tabular}


Table 2b. Complex analysis of variance of essential oils components in essence in Lemon balm plants that are affected by micronutrients and biofertilizeres.

\begin{tabular}{|c|c|c|c|c|c|c|c|}
\hline \multirow[b]{2}{*}{ Source of variation } & \multirow[b]{2}{*}{ D.F } & Neral & Chavicol & Geraniol & Geranial & E-Caryophyllene & Caryophyllene oxide \\
\hline & & & & & & & \\
\hline Year(Y) & 1 & $0.015^{*}$ & $0.019^{*}$ & $0.0035^{*}$ & $0.0016^{*}$ & $0.0023^{*}$ & $0.0027^{*}$ \\
\hline $\mathrm{R} / \mathrm{Y}$ & 4 & $0.009^{*}$ & $0.012^{*}$ & $0.0021^{*}$ & $0.0008^{*}$ & $0.0014^{*}$ & $0.0017^{*}$ \\
\hline Manganese(Mn) & 2 & $0.012^{*}$ & $0.015^{*}$ & $0.0027^{*}$ & $0.0013^{*}$ & $0.0018^{*}$ & $0.0021^{*}$ \\
\hline Boron (B) & 2 & $0.013^{*}$ & $0.017^{*}$ & $0.0029^{*}$ & $0.0014^{*}$ & $0.0019^{*}$ & $0.0022^{*}$ \\
\hline Nitrogen $(\mathrm{N})$ & 1 & $0.016^{*}$ & $0.021^{*}$ & $0.0034^{*}$ & $0.0017^{*}$ & $0.0025^{*}$ & $0.0028^{*}$ \\
\hline Phosphorus (P) & 1 & $0.017^{*}$ & $0.019^{*}$ & $0.0037^{*}$ & $0.0018^{*}$ & $0.0027^{*}$ & $0.0029^{*}$ \\
\hline $\mathrm{Mn} \times \mathrm{B}$ & 4 & $0.011^{*}$ & $0.013^{*}$ & $0.0022^{*}$ & $0.0012^{*}$ & $0.0015^{*}$ & $0.0018^{*}$ \\
\hline $\mathrm{Mn} \times \mathrm{N}$ & 2 & $0.024^{* *}$ & $0.041^{* *}$ & $0.0031^{*}$ & $0.0025^{* *}$ & $0.0036^{* *}$ & $0.0045^{* *}$ \\
\hline $\mathrm{Mn} \times \mathrm{P}$ & 2 & $0.028^{* *}$ & $0.048^{* *}$ & $0.0029^{*}$ & $0.0029^{* *}$ & $0.0037^{* *}$ & $0.0047^{* *}$ \\
\hline $\mathrm{B} \times \mathrm{N}$ & 2 & $0.029^{* *}$ & $0.056^{* *}$ & $0.006^{* *}$ & $0.0031^{* *}$ & $0.0041^{* *}$ & $0.0051^{* *}$ \\
\hline $\mathrm{B} \times \mathrm{P}$ & 2 & $0.032^{* *}$ & $0.066^{* *}$ & $0.0072^{* *}$ & $0.0034^{* *}$ & $0.0045^{* *}$ & $0.0054^{* * *}$ \\
\hline $\mathrm{N} \times \mathrm{P}$ & 1 & $0.036^{* *}$ & $0.043^{* *}$ & $0.0081^{* *}$ & $0.0037^{* *}$ & $0.0024^{*}$ & $0.0027^{*}$ \\
\hline $\mathrm{Mn} \times \mathrm{B} \times \mathrm{N}$ & 4 & $0.022^{* *}$ & $0.012^{*}$ & $0.0024^{*}$ & $0.0024^{* *}$ & $0.0035^{* *}$ & $0.0039^{* *}$ \\
\hline $\mathrm{Mn} \times \mathrm{B} \times \mathrm{P}$ & 4 & $0.032^{* *}$ & $0.044^{* *}$ & $0.004^{* *}$ & $0.0037^{* *}$ & $0.0043^{* *}$ & $0.0041^{* *}$ \\
\hline $\mathrm{Mn} \times \mathrm{N} \times \mathrm{P}$ & 2 & $0.061^{* *}$ & $0.081^{* *}$ & $0.0081^{* *}$ & $0.0065^{*}$ & $0.0056^{* *}$ & $0.0061^{* *}$ \\
\hline $\mathrm{B} \times \mathrm{N} \times \mathrm{P}$ & 2 & $0.055^{* *}$ & $0.092^{* *}$ & $0.0076^{* *}$ & $0.006^{* *}$ & $0.0045^{* *}$ & $0.0055^{* *}$ \\
\hline $\mathrm{Mn} \times \mathrm{B} \times \mathrm{N} \times \mathrm{P}$ & 4 & $0.034^{* *}$ & $0.051^{* *}$ & $0.0032^{* *}$ & $0.0044^{* *}$ & $0.0061^{* *}$ & $0.0044^{* *}$ \\
\hline $\mathrm{T}(\mathrm{Mn} \times \mathrm{B} \times \mathrm{N} \times \mathrm{P}) \times \mathrm{Y}$ & 35 & $0.0061^{*}$ & $0.0075^{*}$ & $0.0014^{*}$ & $0.00062^{*}$ & $0.00091^{*}$ & $0.001^{*}$ \\
\hline $\mathrm{E}$ & 140 & 0.004 & 0.005 & 0.0009 & 0.0004 & 0.0006 & 0.0007 \\
\hline Coefficient of vari & & 1.5 & 1.2 & 2.4 & 1.1 & 1.5 & 1.1 \\
\hline
\end{tabular}

Biofertilizeres by $\mathrm{H}_{2} \mathrm{Bo}^{3-}$ and $\mathrm{Mn}^{2+}$ applications affected geranial, geraniol and geranyl acetate of stems and increased with the micro/macronutrients applications. There were statistical significant differences among the three rates of $\mathrm{H}_{2} \mathrm{Bo}^{3-}$ and $\mathrm{Mn}^{2+}$ applications and application or/no application of biofertilizeres (Table 2). The amount of geranial per plant increased after the fertilizers application compared with the control by an average of (12\%) (Tables 3, 5, 6).

Linalool and neral concentrations were affected by biofertilizeres with $\mathrm{H}_{2} \mathrm{Bo}^{3-}$ and $\mathrm{Mn}^{2+}$ applications and therefore increased in various range compared with the control (Tables 3-6). In generally the most of Caryophyllene oxide (8.4\%), E-Caryophyllene (6.9\%), Geranial (33.36\%) and Chavicol (12.38\%) were made by treatments that had biofertilizeres with $150 \mathrm{ppm}$ of $\mathrm{Mn}$ and $\mathrm{B}$. In most of other subsidiary components contain of Linalool, exo-Isocitral, Citronellal, Z-Isoicitral, Rosefuran epoxide, E-Isoicitral, Piperitenone oxide, E-Caryophyllene, alpha-transBergamotene, alpha-Humulene, Germacrene D, Ebeta- Ionone, gamma-Cadinene, Humulene epoxide II, epi-alpha-Cadinol, 14-hydroxy-Z-Caryophyllene, Hexadecanoic acid and Abieta-8 (14),13 (15-diene); the treatments that had micronutrients with biofertilizeres made most of components and in these treatments the treatment of $\mathrm{Mn} 150 \mathrm{ppm} \times \mathrm{B} 150 \mathrm{ppm}$ with use of biofertilizeres was the best. Biofertilizeres with micronutrients applications affected on chavicol percentage of the crop (Tables 3-6). 
Table 3a. Means of essential oil percentage measured in lemon balm plants that are affected by micronutrients $(150 \mathrm{ppm})$ concentration and biofertilizeres $\left(1^{\text {st }}\right.$ year $)$.

\begin{tabular}{|c|c|c|c|c|c|c|c|c|c|c|c|}
\hline Treatments & $\begin{array}{l}\text { 6-methyl-5- } \\
\text { Hepten-2-1 }\end{array}$ & Linalool & exo-Isocitral & Citronellal & Z-Isoicitral & $\begin{array}{c}\text { Rosefuran } \\
\text { epoxide }\end{array}$ & E-Isoicitral & $\begin{array}{c}\text { Methyl } \\
\text { chavicol }\end{array}$ & Neral & Chavicol & E-Caryophylle \\
\hline Manganese(Mn) & $1.43 b$ & $0.45 e f$ & $0.22 b$ & - & $1.15 b c$ & $0.29 c$ & $1.73 b$ & $0.73 d$ & $24.09 b$ & - & $7.54 a$ \\
\hline Boron (B) & $0.42 e$ & $0.44 f$ & $0.22 b$ & $0.37 c$ & $1.32 b$ & - & $1.95 a b$ & - & $19.05 b c$ & $7.33 b$ & $7.17 a$ \\
\hline Nitrogen $(\mathrm{N})$ & $1.36 b c$ & $0.49 e f$ & $0.22 b$ & $0.4 c$ & $1.27 b$ & $0.5 a$ & $1.8 b$ & $0.58 d$ & $14.14 c$ & $12.28 a$ & $7.37 a$ \\
\hline Phosphorus (P) & $1.06 d$ & $0.44 f$ & - & $0.42 b c$ & $1.16 b c$ & $0.39 c$ & $1.65 b c$ & - & $27.83 a$ & - & $5.91 b$ \\
\hline $\mathrm{Mn} \times \mathrm{B}$ & $1.26 c$ & $0.47 e f$ & $0.28 a$ & $0.5 b$ & $1.59 a$ & $0.53 a$ & $2.03 a$ & $2.82 b$ & $14.57 c$ & $12.65 a$ & $6.37 a b$ \\
\hline $\mathrm{Mn} \times \mathrm{N}$ & $1.31 c$ & 0.56 de & - & $0.41 c$ & $1.08 c$ & $0.39 b$ & $1.56 b c$ & $2.01 b$ & $17.46 c$ & $6.16 b$ & $7.38 a$ \\
\hline $\mathrm{Mn} \times \mathrm{P}$ & $1.19 c d$ & $0.39 g$ & $0.21 b$ & $0.35 c$ & $1.1 c$ & $0.4 c$ & $1.54 c$ & $4.1 a$ & $26.25 a$ & - & $7.1 a$ \\
\hline $\mathrm{B} \times \mathrm{N}$ & $1.72 a$ & $0.25 h$ & $0.22 b$ & $0.56 a b$ & $1.28 b$ & $0.45 b c$ & $1.89 b$ & $4.31 a$ & $24.61 b$ & - & $6.09 b$ \\
\hline $\mathrm{B} \times \mathrm{P}$ & $1.26 c$ & $0.48 e f$ & $0.23 b$ & $0.45 b c$ & $1.18 b c$ & $0.46 b c$ & $1.69 b c$ & $1.15 \mathrm{c}$ & $25.59 a b$ & - & $7.35 a$ \\
\hline $\mathrm{N} \times \mathrm{P}$ & $1.37 b c$ & $0.51 e$ & $0.2 b$ & $0.41 c$ & $1.29 b$ & $0.51 a$ & $1.7 b$ & $0.59 d$ & $14.4 c$ & $12.38 a$ & $7.16 a$ \\
\hline $\mathrm{Mn} \times \mathrm{B} \times \mathrm{N}$ & $1.16 d$ & $0.54 e$ & - & $0.62 a$ & $1.16 b c$ & $0.59 a$ & $1.6 b c$ & - & $27.8 a$ & - & $6.1 a b$ \\
\hline $\mathrm{Mn} \times \mathrm{B} \times \mathrm{P}$ & $1.6 a$ & $0.64 d$ & - & $0.2 d$ & $1.32 b$ & $0.6 a$ & $2.05 a$ & - & $19.8 a b$ & - & $5.1 c$ \\
\hline $\mathrm{Mn} \times \mathrm{N} \times \mathrm{P}$ & $1.46 b$ & $0.74 c$ & - & $0.4 c$ & $1.36 b$ & $0.35 c$ & $1.75 b$ & - & $19.9 a b$ & - & $7.6 a$ \\
\hline $\mathrm{B} \times \mathrm{N} \times \mathrm{P}$ & $1.56 a b$ & $0.84 b$ & - & $0.46 b c$ & $1.45 a b$ & $0.37 c$ & $1.05 d$ & - & $18.8 b$ & - & $6.6 a b$ \\
\hline $\mathrm{Mn} \times \mathrm{B} \times \mathrm{N} \times \mathrm{P}$ & $1.32 c$ & $0.94 a$ & - & $0.56 a b$ & $1.56 a$ & $0.59 a$ & $2.6 a$ & - & $19.5 a b$ & - & $6.8 a b$ \\
\hline Control & $0.06 f$ & $0.44 b$ & - & $0.49 b c$ & $1.23 b$ & $0.45 b c$ & $1.65 b c$ & - & $20.3 a b$ & - & $5.16 c$ \\
\hline
\end{tabular}

Table 3b. Means of essential oil percentage measured in lemon balm plants that are affected by micronutrients $(150 \mathrm{ppm})$ concentration and biofertilizeres $\left(1^{\text {st }}\right.$ year $)$.

\begin{tabular}{|c|c|c|c|c|c|c|c|c|c|c|c|}
\hline Treatments & $\begin{array}{l}\text { alpha-trans- } \\
\text { Bergamotene }\end{array}$ & Piperitone & $\begin{array}{c}\text { Methyl } \\
\text { citronellate }\end{array}$ & Geraniol & $\begin{array}{c}\text { trans- } \\
\text { Piperitone } \\
\text { epoxide }\end{array}$ & Geranial & $\begin{array}{l}\text { Methyl } \\
\text { nerolate }\end{array}$ & Nerylformate & $\begin{array}{l}\text { Methyl } \\
\text { geranate }\end{array}$ & $\begin{array}{c}\text { Piperitenone } \\
\text { oxide }\end{array}$ & $\begin{array}{l}\text { Geranyl } \\
\text { acetate }\end{array}$ \\
\hline Manganese(Mn) & - & $0.57 b$ & - & $1.31 b$ & - & $28.9 b$ & - & - & $0.47 b$ & - & $4.9 c$ \\
\hline Boron (B) & - & $0.64 a$ & $0.46 a$ & $1.44 c$ & - & $20.81 c$ & $5.32 a$ & $6 a$ & $0.72 a$ & - & $7.29 a$ \\
\hline Nitrogen $(\mathrm{N})$ & - & $0.56 b$ & $0.4 a$ & $12.75 a$ & $1.37 a$ & $18.58 \mathrm{c}$ & - & - & $0.49 b$ & - & $5.44 b$ \\
\hline Phosphorus (P) & - & $0.6 a b$ & $0.27 b c$ & $1.2 c$ & - & $32.36 a$ & - & - & - & - & $4.85 b c$ \\
\hline $\mathrm{Mn} \times \mathrm{B}$ & $0.5 b$ & $0.38 c$ & $0.32 b$ & $0.7 c$ & - & $32.49 a$ & - & - & $0.46 b$ & $4.88 a$ & - \\
\hline $\mathrm{Mn} \times \mathrm{N}$ & $1.12 a$ & $0.47 c$ & $0.27 b c$ & $1.05 c$ & - & $27 b$ & - & - & - & - & $5.09 b c$ \\
\hline $\mathrm{Mn} \times \mathrm{P}$ & $0.4 b$ & $0.7 a$ & - & $1.15 c$ & - & $31.39 a$ & - & - & $0.46 b$ & - & $5.31 b$ \\
\hline $\mathrm{B} \times \mathrm{N}$ & $0.28 b$ & $0.39 c$ & - & $0.81 c$ & - & $19.19 c$ & $3.87 b$ & $5.3 a$ & $0.51 b$ & $1.11 b$ & $4.1 c$ \\
\hline $\mathrm{B} \times \mathrm{P}$ & - & $0.47 c$ & $0.23 c$ & $0.77 c$ & - & $30.82 a b$ & - & - & - & $0.55 c$ & $6.07 a b$ \\
\hline $\mathrm{N} \times \mathrm{P}$ & $0.39 b$ & $0.57 b$ & $0.4 a$ & $12.7 a$ & $1.4 a$ & $18.5 c$ & - & $4.6 b$ & $0.44 b$ & - & $3.42 c$ \\
\hline $\mathrm{Mn} \times \mathrm{B} \times \mathrm{N}$ & $1.3 a$ & $0.6 a b$ & $0.27 b c$ & $1.42 c$ & - & $32.36 a$ & - & $1.6 e$ & $0.48 b$ & - & $4.4 c$ \\
\hline $\mathrm{Mn} \times \mathrm{B} \times \mathrm{P}$ & $1.9 a$ & $0.63 a b$ & $0.2 b c$ & $1.6 c$ & - & $32.16 a$ & - & $1.7 e$ & $0.49 b$ & - & $3.5 c$ \\
\hline $\mathrm{Mn} \times \mathrm{N} \times \mathrm{P}$ & $1.9 a$ & $0.45 c$ & $0.37 b$ & $1.8 \mathrm{c}$ & - & $32.36 a$ & - & $2.6 d$ & $0.54 b$ & - & $7.4 a$ \\
\hline $\mathrm{B} \times \mathrm{N} \times \mathrm{P}$ & $1.7 a$ & $0.56 b$ & $0.22 c$ & $7.9 b$ & - & $32.6 a$ & - & $2.6 d$ & $0.64 a$ & - & $5.6 b$ \\
\hline $\mathrm{Mn} \times \mathrm{B} \times \mathrm{N} \times \mathrm{P}$ & $1.9 a$ & $0.5 b$ & $0.23 c$ & $8.2 b$ & - & $33.36 a$ & - & $2.2 d$ & $0.4 b$ & - & $3.4 c$ \\
\hline Control & $0.29 b$ & $0.66 a$ & $0.29 b$ & $9.2 a b$ & - & $31.16 a$ & - & $3.6 c$ & $0.14 c$ & - & $3.4 c$ \\
\hline
\end{tabular}

Table 3c. Means of essential oil percentage measured in lemon balm plants that are affected by micronutrients $(150 \mathrm{ppm})$ concentration and biofertilizeres ( $1^{\text {st }}$ year$)$.

\begin{tabular}{|c|c|c|c|c|c|c|c|c|c|c|c|}
\hline Treatments & $\begin{array}{c}\text { alpha- } \\
\text { Humulene }\end{array}$ & Germacrene D & $\begin{array}{l}\text { E-beta- } \\
\text { Ionone }\end{array}$ & $\begin{array}{l}\text { gamma- } \\
\text { Cadinene }\end{array}$ & $\begin{array}{l}\text { Caryophyllene } \\
\text { oxide }\end{array}$ & $\begin{array}{l}\text { Humulene } \\
\text { epoxide II }\end{array}$ & $\begin{array}{c}\text { epic- } \\
\text { alpha- } \\
\text { Cadinol }\end{array}$ & $\begin{array}{l}\text { 14-hydroxy-Z- } \\
\text { Caryophyllene }\end{array}$ & $\begin{array}{l}\text { Hexadecanoic } \\
\text { acid }\end{array}$ & $\begin{array}{c}\text { Abieta- } \\
8(14), 13(15- \\
\text { diene })\end{array}$ & $\begin{array}{c}\text { Total } \\
\%\end{array}$ \\
\hline Manganese(Mn) & $0.52 b c$ & - & - & - & $12.46 a$ & $0.72 a$ & - & $0.74 a$ & $0.29 a b$ & $2.59 a$ & 91.1 \\
\hline Boron (B) & $0.47 c$ & - & $0.24 b$ & - & $9.79 b c$ & $0.49 b$ & - & $0.6 b$ & - & $1.75 b$ & 94.3 \\
\hline Nitrogen $(\mathrm{N})$ & - & - & $0.24 b$ & - & $11.29 a b$ & - & - & $0.65 a b$ & - & $2.19 a$ & 94.36 \\
\hline Phosphorus $(\mathrm{P})$ & $0.39 c$ & & $0.22 b$ & - & $10.98 b$ & $0.56 b$ & & $0.61 b$ & - & $2.11 a$ & 93.01 \\
\hline $\mathrm{Mn} \times \mathrm{B}$ & $1.59 a$ & $0.93 a$ & $0.3 a b$ & $1.46 a$ & $6.37 d$ & - & $0.4 b$ & $0.39 c$ & $0.24 b$ & - & 94.49 \\
\hline $\mathrm{Mn} \times \mathrm{N}$ & $0.71 b$ & $0.35 b$ & $0.42 a$ & $0.7 b$ & $12.62 a$ & $0.64 a b$ & $1.35 a$ & $0.64 a b$ & $0.26 b$ & $1.59 b$ & 92.6 \\
\hline $\mathrm{Mn} \times \mathrm{P}$ & $1.29 a$ & $0.57 b$ & $0.3 a b$ & $1.12 a b$ & $8.13 c$ & $0.51 b$ & $0.23 b$ & $0.46 c$ & - & $1.22 b$ & 95.89 \\
\hline $\mathrm{B} \times \mathrm{N}$ & $0.88 a b$ & $0.4 b$ & $0.28 b$ & - & $9.89 b c$ & $0.54 b$ & $0.29 b$ & $0.71 a$ & - & $1.34 b$ & 91.24 \\
\hline$B \times P$ & $0.55 b$ & - & $0.23 b$ & - & $11.48 a b$ & $0.61 a b$ & - & $0.64 a b$ & - & $2.04 a b$ & 94.3 \\
\hline $\mathrm{N} \times \mathrm{P}$ & $1.5 a$ & - & $0.3 a b$ & - & $10.6 b$ & $0.2 c$ & $1.2 a$ & - & $0.36 a$ & $1.3 b$ & 98.4 \\
\hline $\mathrm{Mn} \times \mathrm{B} \times \mathrm{N}$ & $1.6 a$ & - & $0.31 a b$ & - & $8.4 c$ & $0.7 a$ & $1.3 a$ & - & $0.3 a b$ & $1.64 b$ & 96.25 \\
\hline $\mathrm{Mn} \times \mathrm{B} \times \mathrm{P}$ & $1.35 a$ & - & $0.32 a b$ & - & $12.3 a$ & $0.34 c$ & $1.02 a$ & - & $0.3 a b$ & $2.34 a$ & 91.46 \\
\hline $\mathrm{Mn} \times \mathrm{N} \times \mathrm{P}$ & $1.5 a$ & - & $0.34 a b$ & - & $8.6 c$ & $0.77 a$ & $1.12 a$ & - & $0.32 a b$ & $2.64 a$ & 96.27 \\
\hline $\mathrm{B} \times \mathrm{N} \times \mathrm{P}$ & $1.6 a$ & - & $0.35 a b$ & - & $6.6 d$ & $0.7 a$ & $1.42 a$ & - & $0.4 a$ & $1.54 b$ & 95.56 \\
\hline $\mathrm{Mn} \times \mathrm{B} \times \mathrm{N} \times \mathrm{P}$ & $1.65 a$ & - & $0.38 a b$ & - & $8.6 c$ & $0.57 b$ & $1.65 a$ & - & $0.42 a$ & $2.64 a$ & 94.69 \\
\hline Control & $0.65 b$ & - & $0.3 a b$ & - & $8.6 c$ & $0.5 b$ & $1.2 a$ & - & $0.3 a b$ & $1.34 b$ & 91.41 \\
\hline
\end{tabular}


Table 4a. Means of essential oil percentage measured in lemon balm plants that are affected by micronutrients $(150 \mathrm{ppm})$ concentration and biofertilizeres $\left(2^{\text {nd }}\right.$ year $)$.

\begin{tabular}{|c|c|c|c|c|c|c|c|c|c|c|c|}
\hline Treatments & $\begin{array}{l}\text { alpha-trans- } \\
\text { Bergamotene }\end{array}$ & $\begin{array}{l}\text { 6-methyl- } \\
\text { 5-Hepten- } \\
\text { 2-one }\end{array}$ & Linalool & $\begin{array}{c}\text { exo- } \\
\text { Isocitral }\end{array}$ & Citronellal & $\begin{array}{c}\mathrm{Z}- \\
\text { Isoicitral }\end{array}$ & $\begin{array}{c}\text { Rosefuran } \\
\text { epoxide }\end{array}$ & $\begin{array}{c}\text { E- } \\
\text { Isoicitral }\end{array}$ & $\begin{array}{l}\text { Methyl } \\
\text { chavicol }\end{array}$ & Geranial & $\begin{array}{l}\text { Methyl } \\
\text { nerolate }\end{array}$ \\
\hline Manganese(Mn) & $1.77 b$ & $1.3 c$ & $0.43 c$ & $0.34 a$ & $0.43 b$ & $1.6 a$ & $0.52 a b$ & $1.82 b$ & $0.68 d$ & $19.1 c$ & $1.77 c$ \\
\hline Boron (B) & $1.23 c$ & $0.62 e$ & $0.46 c$ & $0.34 a$ & $0.39 b$ & $1.4 a b$ & - & $1.86 b$ & - & $21.8 b c$ & $4.32 a$ \\
\hline Nitrogen (N) & - & $1.4 b$ & $0.55 c$ & $0.33 a$ & - & $1.21 c$ & $0.17 d$ & $1.7 b c$ & $0.6 d$ & $30.19 a b$ & - \\
\hline Phosphorus (P) & - & $1.6 a$ & $0.64 b c$ & - & $0.44 b$ & $1.41 a b$ & $0.43 b$ & $1.85 b$ & - & $35.6 a$ & - \\
\hline $\mathrm{Mn} \times \mathrm{B}$ & $0.5 d$ & $1.2 c d$ & $0.77 a b$ & $0.34 a$ & $0.51 a b$ & $1.59 a$ & $0.55 a b$ & $1.9 b$ & $2.8 \mathrm{c}$ & $31.49 a$ & $1.75 c$ \\
\hline $\mathrm{Mn} \times \mathrm{N}$ & $1.12 c$ & $1.31 \mathrm{c}$ & $0.56 c$ & - & $0.41 b$ & $1.08 c$ & $0.44 b$ & $1.54 c$ & $2.1 c$ & $27.3 b$ & - \\
\hline $\mathrm{Mn} \times \mathrm{P}$ & $0.4 d$ & $1.19 c d$ & $0.39 c d$ & $0.21 b$ & $0.35 d$ & $1.1 c$ & $0.41 b$ & $1.56 c$ & $7.01 a$ & $32.3 a$ & - \\
\hline $\mathrm{B} \times \mathrm{N}$ & $0.28 d$ & $1.72 a$ & $0.25 d$ & $0.22 b$ & $0.56 a$ & $1.28 b$ & $0.45 b$ & $1.89 a b$ & $4.31 b$ & $21.1 b c$ & $3.87 a$ \\
\hline $\mathrm{B} \times \mathrm{P}$ & - & $1.26 \mathrm{~cd}$ & $0.48 c$ & $0.23 b$ & $0.45 b$ & $1.18 c$ & $0.46 b$ & $1.6 b c$ & $1.15 c$ & $29.8 b$ & - \\
\hline $\mathrm{N} \times \mathrm{P}$ & $0.39 d$ & $1.3 c$ & $0.5 c$ & $0.2 b$ & $0.4 b$ & $1.29 b$ & $0.51 a b$ & $1.7 b c$ & $0.59 d$ & $21.5 b c$ & $2.7 b$ \\
\hline $\mathrm{Mn} \times \mathrm{B} \times \mathrm{N}$ & $1.3 c$ & $1.12 d$ & $0.54 c$ & - & $0.6 a$ & $1.16 c$ & $0.69 a$ & $1.64 b c$ & - & $31.3 a b$ & $3.37 a b$ \\
\hline $\mathrm{Mn} \times \mathrm{B} \times \mathrm{P}$ & $1.9 b$ & $1.16 d$ & $0.5 c$ & - & $0.62 a$ & $1.6 a$ & $0.59 a b$ & $1.6 b c$ & - & $31.3 a b$ & $1.87 c$ \\
\hline $\mathrm{Mn} \times \mathrm{N} \times \mathrm{P}$ & $1.8 b$ & $1.6 a$ & $0.6 b c$ & $0.2 b$ & $0.23 d$ & $1.39 a b$ & $0.66 a$ & $2.25 a$ & $0.55 d$ & $32.6 a$ & $3.5 a b$ \\
\hline $\mathrm{B} \times \mathrm{N} \times \mathrm{P}$ & $1.8 b$ & $1.4 b$ & $0.74 b$ & - & $0.48 b$ & $1.3 b$ & $0.35 c$ & $1.65 b c$ & $0.69 d$ & $28.6 b$ & $1.77 c$ \\
\hline $\mathrm{Mn} \times \mathrm{B} \times \mathrm{N} \times \mathrm{P}$ & $2.1 a$ & $1.5 a b$ & $0.86 a$ & - & $0.7 a$ & $1.6 a$ & $0.58 a b$ & $2.4 a$ & - & $31.6 a b$ & $1.77 c$ \\
\hline Control & $1.9 b$ & $1.3 c$ & $0.7 b$ & - & $0.45 b$ & $1.5 a$ & $0.39 c$ & $1.15 d$ & - & $33.56 a$ & - \\
\hline
\end{tabular}

Table 4b. Means of essential oil percentage measured in lemon balm plants that are affected by micronutrients $(150 \mathrm{ppm})$ concentration and biofertilizeres $\left(2^{\text {nd }}\right.$ year $)$.

\begin{tabular}{|c|c|c|c|c|c|c|c|c|c|c|c|}
\hline Treatments & Neral & Chavicol & Piperitone & $\begin{array}{c}\text { Methyl } \\
\text { citronellate }\end{array}$ & Geraniol & $\begin{array}{c}\text { trans- } \\
\text { Piperitone } \\
\text { epoxide }\end{array}$ & Nerylformate & $\begin{array}{l}\text { Methyl } \\
\text { geranate }\end{array}$ & $\begin{array}{l}\text { Piperitenone } \\
\text { oxide }\end{array}$ & $\begin{array}{c}\text { Geranyl } \\
\text { acetate }\end{array}$ & E-Caryophyllene \\
\hline Manganese(Mn) & $14.3 d$ & $12.2 a$ & $0.59 b$ & $0.43 a$ & $2.75 b$ & $1.77 a$ & - & $0.37 c$ & $1.77 b$ & $4.34 b c$ & $5.5 b$ \\
\hline Boron (B) & $18.5 c$ & $8.3 b$ & $0.68 a b$ & $0.49 a$ & $1.84 c$ & $1.41 a$ & $6.2 a$ & $0.7 a$ & - & $7.2 a$ & $7.1 a$ \\
\hline Nitrogen $(\mathrm{N})$ & $24.9 a b$ & - & $0.59 b$ & - & $1.71 \mathrm{c}$ & $1.47 a$ & - & $0.21 c$ & $1.47 b$ & $5.4 b$ & $7.17 a$ \\
\hline Phosphorus (P) & $25.3 a$ & - & $0.67 a b$ & $0.47 a$ & $1.23 c$ & - & - & - & - & $4.8 b c$ & $5.9 b$ \\
\hline $\mathrm{Mn} \times \mathrm{B}$ & $15.5 d$ & $9.9 b$ & $0.42 d$ & $0.42 a$ & $0.77 d$ & - & $1.7 b$ & $0.4 b$ & $4.8 a$ & - & $6.3 a b$ \\
\hline $\mathrm{Mn} \times \mathrm{N}$ & $18.4 c$ & $6.6 c$ & $0.49 d$ & $0.47 a$ & $1.5 c$ & - & - & - & - & $5.2 b$ & $7.3 a$ \\
\hline $\mathrm{Mn} \times \mathrm{P}$ & $22.25 b$ & - & $0.71 a$ & - & $1.85 c$ & - & - & $0.46 b$ & - & $5.3 b$ & $7.11 a$ \\
\hline $\mathrm{B} \times \mathrm{N}$ & $24.61 a b$ & - & $0.39 d$ & - & $0.81 d$ & - & $5.3 a$ & $0.51 b$ & $1.11 b$ & $4.1 b c$ & $6.09 a b$ \\
\hline $\mathrm{B} \times \mathrm{P}$ & $25.59 a$ & - & $0.47 d$ & $0.25 a$ & $0.77 d$ & - & - & - & $0.55 c$ & $6.7 a$ & $7.35 a$ \\
\hline $\mathrm{N} \times \mathrm{P}$ & $14.2 d$ & $12.38 a$ & $0.57 b$ & $0.44 a$ & $2.7 b$ & $1.4 a$ & $5.6 a$ & $0.44 b$ & - & $3.4 c$ & $7.16 a$ \\
\hline $\mathrm{Mn} \times \mathrm{B} \times \mathrm{N}$ & $26.8 a$ & - & $0.6 a b$ & $0.27 b$ & $1.4 c$ & - & $2.6 b$ & $0.48 b$ & - & $4.1 b c$ & $6.1 a b$ \\
\hline $\mathrm{Mn} \times \mathrm{B} \times \mathrm{P}$ & $25.8 a$ & - & $0.61 a b$ & $0.27 b$ & $1.32 c$ & $1.2 a b$ & $1.9 b$ & $0.79 a$ & - & $3.4 c$ & $5.1 b$ \\
\hline $\mathrm{Mn} \times \mathrm{N} \times \mathrm{P}$ & $13.7 d$ & - & $0.62 a b$ & $0.27 b$ & $1.26 c$ & $1.4 a$ & $2.5 b$ & $0.5 b$ & - & $7.34 a$ & $7.7 a$ \\
\hline $\mathrm{B} \times \mathrm{N} \times \mathrm{P}$ & $15.6 d$ & $6.6 c$ & $0.35 d$ & $0.47 a$ & $1.28 c$ & $1.77 a$ & $2.4 b$ & $0.6 a$ & - & $5.9 b$ & $6.9 a b$ \\
\hline $\mathrm{Mn} \times \mathrm{B} \times \mathrm{N} \times \mathrm{P}$ & $18.4 c$ & $2.16 d$ & $0.54 b$ & $0.22 b$ & $2.9 b$ & $1.87 a$ & $2.9 b$ & $0.63 a$ & - & $3.1 c$ & $6.9 a b$ \\
\hline Control & $19.2 c$ & - & $0.5 b$ & $0.23 b$ & $8.1 a$ & $0.9 b$ & $1.6 b$ & $0.72 a$ & - & $3.6 c$ & $6.5 a b$ \\
\hline
\end{tabular}

Table 4c. Means of essential oil percentage measured in lemon balm plants that are affected by micronutrients $(150 \mathrm{ppm})$ concentration and biofertilizeres $\left(2^{\text {nd }}\right.$ year $)$.

\begin{tabular}{|c|c|c|c|c|c|c|c|c|c|c|c|}
\hline Treatments & $\begin{array}{l}\text { alpha- } \\
\text { Humulene }\end{array}$ & Germacrene D & $\begin{array}{l}\text { E-beta- } \\
\text { Ionone }\end{array}$ & $\begin{array}{l}\text { gamma- } \\
\text { Cadinene }\end{array}$ & $\begin{array}{l}\text { Caryophyllene } \\
\text { oxide }\end{array}$ & $\begin{array}{l}\text { Humulene } \\
\text { epoxide II }\end{array}$ & $\begin{array}{c}\text { epi- } \\
\text { alpha- } \\
\text { Cadinol } \\
\end{array}$ & $\begin{array}{l}\text { 14-hydroxy-Z- } \\
\text { Caryophyllene }\end{array}$ & $\begin{array}{l}\text { Hexadecanoic } \\
\text { acid }\end{array}$ & $\begin{array}{c}\text { Abieta- } \\
\text { 8(14),13(15- } \\
\text { diene) } \\
\end{array}$ & $\begin{array}{c}\text { Total } \\
\%\end{array}$ \\
\hline Manganese(Mn) & $0.5 d$ & $1.37 a$ & $0.7 a$ & $1.77 a$ & $11.4 a$ & $0.7 a$ & $0.77 b$ & $0.44 b$ & $0.33 b$ & $2.5 a b$ & 94.26 \\
\hline Boron (B) & $0.4 d$ & - & $0.14 c$ & - & $9.7 b c$ & $0.4 b$ & - & $0.62 a$ & $0.37 b$ & $1.7 b c$ & 98.17 \\
\hline Nitrogen $(\mathrm{N})$ & - & - & $0.2 c$ & - & $10.2 b$ & - & - & $0.61 a$ & - & $2.1 b$ & 92.18 \\
\hline Phosphorus (P) & $0.44 d$ & - & $0.2 c$ & - & $10.9 b$ & $0.5 b$ & - & $0.6 a$ & - & $3.1 a$ & 96.08 \\
\hline $\mathrm{Mn} \times \mathrm{B}$ & $1.5 b$ & $0.9 b$ & $0.12 c$ & $1.4 a$ & $6.3 d$ & - & $0.41 c$ & $0.44 b$ & $0.64 a$ & - & 95.32 \\
\hline $\mathrm{Mn} \times \mathrm{N}$ & $0.7 d$ & $0.45 c$ & $0.4 b$ & $0.72 b$ & $12.6 a$ & $0.14 c$ & $1.3 a b$ & $0.54 a b$ & $0.26 b$ & $1.5 b c$ & 94.43 \\
\hline $\mathrm{Mn} \times \mathrm{P}$ & $1.3 c$ & $0.67 b c$ & $0.2 c$ & $1.1 a b$ & $8.1 c$ & $0.5 b$ & $0.2 c$ & $0.4 b$ & - & $1.2 c$ & 96.27 \\
\hline $\mathrm{B} \times \mathrm{N}$ & $0.88 d$ & $0.42 c$ & $0.28 c$ & - & $9.8 b c$ & $0.5 b$ & $0.29 c$ & $0.7 a$ & - & $1.4 b c$ & 93.12 \\
\hline$B \times P$ & $0.55 d$ & - & $0.23 c$ & - & $11.4 a$ & $0.61 a b$ & - & $0.64 a$ & - & $2.4 b$ & 94.12 \\
\hline $\mathrm{N} \times \mathrm{P}$ & $1.5 b$ & - & $0.3 b$ & - & $10.6 b$ & $0.22 c$ & $1.1 a b$ & - & $0.36 b$ & $1.3 b c$ & 94.75 \\
\hline $\mathrm{Mn} \times \mathrm{B} \times \mathrm{N}$ & $1.65 a b$ & - & $0.41 b$ & - & $6.4 d$ & $0.71 a$ & $1.2 a b$ & - & $0.3 b$ & $1.6 b c$ & 96.34 \\
\hline $\mathrm{Mn} \times \mathrm{B} \times \mathrm{P}$ & $1.45 b$ & - & $0.42 b$ & - & $8.3 c$ & $0.44 b$ & $1.2 a b$ & - & $0.31 b$ & $2.3 b$ & 95.95 \\
\hline $\mathrm{Mn} \times \mathrm{N} \times \mathrm{P}$ & $1.53 b$ & - & $0.44 b$ & - & $8.4 c$ & $0.74 a$ & $1.1 a b$ & - & $0.32 b$ & $2.6 a b$ & 95.8 \\
\hline$B \times N \times P$ & $1.66 a b$ & - & $0.45 b$ & - & $6.3 d$ & $0.71 a$ & $1.4 a$ & - & $0.43 a b$ & $2.5 a b$ & 96.1 \\
\hline $\mathrm{Mn} \times \mathrm{B} \times \mathrm{N} \times \mathrm{P}$ & $1.66 a b$ & - & $0.48 b$ & - & $8.4 c$ & $0.5 b$ & $1.6 a$ & - & $0.64 a$ & $2.6 a b$ & 99.15 \\
\hline Control & $0.8 a$ & - & $0.44 b$ & - & $6.2 d$ & $0.6 a b$ & $1.4 a$ & - & $0.41 a b$ & $1.5 b c$ & 93.65 \\
\hline
\end{tabular}


Table 5a. Means of essential oil percentage measured in lemon balm plants that are affected by micronutrients (300 ppm) concentration and biofertilizeres $\left(1^{\text {st }}\right.$ year $)$.

\begin{tabular}{|c|c|c|c|c|c|c|c|c|c|c|c|}
\hline Treatments & $\begin{array}{l}\text { 6-methyl-5- } \\
\text { Hepten-2- } \\
\text { one }\end{array}$ & Linalool & exo-Isocitral & Citronellal & Z-Isoicitral & $\begin{array}{c}\text { Rosefuran } \\
\text { epoxide }\end{array}$ & E-Isoicitral & $\begin{array}{c}\text { Methyl } \\
\text { chavicol }\end{array}$ & Neral & Chavicol & Piperitone \\
\hline Manganese(Mn) & $1.62 a$ & $0.46 d$ & $0.34 a$ & $0.39 b$ & $1.4 a$ & - & $1.86 b$ & - & $15.5 c d$ & $8.3 a$ & $0.8 b$ \\
\hline Boron (B) & $1.4 b$ & $0.54 c d$ & $0.34 a$ & - & $1.21 a$ & $0.57 b$ & $1.75 b$ & $0.65 d$ & $24.5 a b$ & - & $0.5 c$ \\
\hline Nitrogen $(\mathrm{N})$ & $1.4 b$ & $0.64 b$ & - & $0.43 b$ & $1.43 a$ & $0.53 b$ & $1.8 a b$ & - & $25.5 a$ & - & $0.67 b c$ \\
\hline Phosphorus $(\mathrm{P})$ & $1.24 c$ & $0.74 b$ & $0.34 a$ & $0.51 b$ & $1.53 a$ & $0.55 b$ & $1.95 a b$ & $2.58 b c$ & $15.5 \mathrm{~cd}$ & $9.9 a$ & $0.42 c$ \\
\hline $\mathrm{Mn} \times \mathrm{B}$ & $1.41 b$ & $0.56 c d$ & - & $0.41 b$ & $1.38 a$ & $0.44 b$ & $1.5 b c$ & $2.1 c$ & $18.4 c$ & $6.6 b$ & $0.4 c$ \\
\hline $\mathrm{Mn} \times \mathrm{N}$ & $1.14 c$ & $0.39 d$ & $0.41 a$ & $0.33 b$ & $1.3 a$ & $0.45 b$ & $1.55 b$ & $7.51 a$ & $22.2 b$ & - & $0.71 b$ \\
\hline $\mathrm{Mn} \times \mathrm{P}$ & $1.14 c$ & $0.39 d$ & $0.24 b$ & $0.33 b$ & $1.3 a$ & $0.54 b$ & $1.54 b$ & $4.15 b$ & $19.5 b c$ & - & $0.75 b$ \\
\hline $\mathrm{B} \times \mathrm{N}$ & $1.42 b$ & $0.45 d$ & $0.24 b$ & $0.53 b$ & $1.38 a$ & $0.45 b$ & $1.59 b$ & $4.31 b$ & $24.1 a b$ & - & $0.39 c$ \\
\hline $\mathrm{B} \times \mathrm{P}$ & $1.46 b$ & $0.48 d$ & $0.23 b$ & $0.43 b$ & $1.38 a$ & $0.45 b$ & $1.69 b$ & $1.1 c$ & $25.59 a$ & - & $0.4 c$ \\
\hline $\mathrm{N} \times \mathrm{P}$ & $1.3 b c$ & $0.54 c d$ & $0.2 b$ & $0.43 b$ & $1.29 a$ & $0.51 b$ & $1.7 b$ & $0.5 d$ & $14.1 d$ & $2.3 c$ & $0.5 c$ \\
\hline $\mathrm{Mn} \times \mathrm{B} \times \mathrm{N}$ & $1.12 c$ & $0.54 c d$ & - & $0.3 b$ & $1.13 a$ & $0.65 b$ & $1.64 b$ & - & $26.8 a$ & - & $0.65 b c$ \\
\hline $\mathrm{Mn} \times \mathrm{B} \times \mathrm{P}$ & $1.46 b$ & $0.4 d$ & - & $0.32 b$ & $1.3 a$ & $0.59 b$ & $1.56 b$ & - & $25.8 a$ & - & $0.61 b c$ \\
\hline $\mathrm{Mn} \times \mathrm{N} \times \mathrm{P}$ & $1.6 a$ & $0.6 c$ & $0.24 b$ & $0.23 b$ & $1.39 a$ & $0.56 b$ & $2.5 a$ & $0.5 d$ & $13.5 d$ & - & $0.6 b c$ \\
\hline $\mathrm{B} \times \mathrm{N} \times \mathrm{P}$ & $1.4 b$ & $0.44 d$ & - & $1.03 a$ & $1.3 a$ & $0.35 b$ & $1.5 b c$ & $0.65 d$ & $15.6 \mathrm{~cd}$ & $6.6 b$ & $0.35 c$ \\
\hline $\mathrm{Mn} \times \mathrm{B} \times \mathrm{N} \times \mathrm{P}$ & $1.2 c$ & $1.4 a$ & $0.2 b$ & $1.3 a$ & $1.2 a$ & $1.33 a$ & $2.1 a$ & - & $18.1 \mathrm{c}$ & $2.1 c$ & $0.5 c$ \\
\hline Control & $1.2 c$ & $0.81 b$ & - & $0.43 b$ & $1.2 a$ & $0.3 b$ & $1.5 b$ & - & $16.2 c$ & - & $1.5 a$ \\
\hline
\end{tabular}

Table 5b. Means of essential oil percentage measured in lemon balm plants that are affected by micronutrients (300 ppm) concentration and biofertilizeres $\left(1^{\text {st }}\right.$ year $)$.

\begin{tabular}{|c|c|c|c|c|c|c|c|c|c|c|c|}
\hline Treatments & $\begin{array}{c}\text { Methyl } \\
\text { citronellate }\end{array}$ & Geraniol & $\begin{array}{l}\text { trans- } \\
\text { Piperitone } \\
\text { epoxide }\end{array}$ & Geranial & $\begin{array}{l}\text { Methyl } \\
\text { nerolate }\end{array}$ & Nerylformate & $\begin{array}{l}\text { Methyl } \\
\text { geranate }\end{array}$ & $\begin{array}{l}\text { Piperitenone } \\
\text { oxide }\end{array}$ & $\begin{array}{l}\text { Geranyl } \\
\text { acetate }\end{array}$ & $\begin{array}{c}\text { E- } \\
\text { Caryophyllene }\end{array}$ & $\begin{array}{l}\text { alpha-trans- } \\
\text { Bergamotene }\end{array}$ \\
\hline Manganese(Mn) & $0.4 b$ & $1.8 b$ & $1.41 a b$ & $21.85 b c$ & $4.3 a$ & - & $0.4 a b$ & - & $5.4 b$ & $7.3 a$ & - \\
\hline Boron (B) & - & $1.7 b$ & $1.45 a b$ & $30.1 a b$ & - & - & - & - & $4.8 b c$ & $5.9 b$ & - \\
\hline Nitrogen $(\mathrm{N})$ & $0.4 b$ & $1.23 b c$ & - & $35.56 a$ & - & - & $0.4 a b$ & $4.8 a$ & - & $6.3 a b$ & $0.5 c$ \\
\hline Phosphorus (P) & $0.42 b$ & $0.7 c$ & - & $31.4 a$ & $1.7 b$ & - & - & - & $5.9 b$ & $7.38 a$ & $1.2 b$ \\
\hline $\mathrm{Mn} \times \mathrm{B}$ & $0.47 b$ & $1.5 b$ & - & $27.35 b$ & - & - & $0.4 a b$ & - & $5.31 b$ & $7.1 a$ & $0.34 b$ \\
\hline $\mathrm{Mn} \times \mathrm{N}$ & - & $1.8 b$ & - & $32.3 a$ & - & - & $0.4 a b$ & - & $5.3 b$ & $7.11 a$ & $0.43 c$ \\
\hline $\mathrm{Mn} \times \mathrm{P}$ & - & $1.15 b c$ & - & $31.3 a$ & - & $5.3 a$ & $0.5 a$ & $1.13 b$ & $4.13 b c$ & $6.9 a b$ & $0.8 c$ \\
\hline $\mathrm{B} \times \mathrm{N}$ & - & $0.8 c$ & - & $19.9 c$ & $3.8 a$ & - & - & $0.53 c$ & $6.73 a$ & $7.35 a$ & - \\
\hline $\mathrm{B} \times \mathrm{P}$ & $0.3 b$ & $0.7 c$ & - & $30.8 a b$ & - & $5.6 a$ & $0.4 a b$ & - & $3.4 c$ & $7.6 a$ & $0.3 c$ \\
\hline$N \times P$ & $0.44 b$ & $2.7 b$ & $1.4 a b$ & $21.5 b c$ & $2.7 a b$ & $5.2 a$ & $0.4 a b$ & - & $3.43 c$ & $7.16 a$ & $0.39 c$ \\
\hline $\mathrm{Mn} \times \mathrm{B} \times \mathrm{N}$ & $0.2 b$ & $1.4 b$ & - & $31.35 a$ & $3.3 a$ & $2.1 b$ & $0.4 a b$ & - & $4.1 b c$ & $6.13 a b$ & $1.33 b$ \\
\hline $\mathrm{Mn} \times \mathrm{B} \times \mathrm{P}$ & $0.27 b$ & $1.3 b$ & $1.2 b$ & $31.3 a$ & $1.8 b$ & $1.2 b$ & $0.39 b$ & - & $3.43 c$ & $5.13 b$ & $1.93 a$ \\
\hline $\mathrm{Mn} \times \mathrm{N} \times \mathrm{P}$ & $0.2 b$ & $1.26 b$ & $1.45 a b$ & $32.65 a$ & $3.5 a$ & $2.2 b$ & $0.53 a$ & - & $7.34 a$ & $7.7 a$ & $1.8 a$ \\
\hline $\mathrm{B} \times \mathrm{N} \times \mathrm{P}$ & $0.4 b$ & $1.2 b$ & $1.7 a$ & $28.6 b$ & $1.7 b$ & $2.2 b$ & $0.63 a$ & - & $5.93 b$ & $6.39 a b$ & $1.8 a$ \\
\hline $\mathrm{Mn} \times \mathrm{B} \times \mathrm{N} \times \mathrm{P}$ & $0.2 b$ & $2.91 b$ & $1.8 a$ & $32.6 a$ & $1.77 b$ & $2.2 b$ & $0.63 a$ & - & $3.1 c$ & $6.93 a b$ & $2.13 a$ \\
\hline Control & $1.2 a$ & $6.5 a$ & $1.95 a$ & $31.5 a$ & - & $1.6 b$ & $0.72 a$ & - & $3.6 c$ & $6.5 a b$ & $1.9 a$ \\
\hline
\end{tabular}

Table 5c. Means of essential oil percentage measured in lemon balm plants that are affected by micronutrients (300 ppm) concentration and biofertilizeres ( $1^{\text {st }}$ year).

\begin{tabular}{|c|c|c|c|c|c|c|c|c|c|c|c|}
\hline Treatments & $\begin{array}{c}\text { alpha- } \\
\text { Humulene }\end{array}$ & Germacrene D & $\begin{array}{l}\text { E-beta- } \\
\text { Ionone }\end{array}$ & $\begin{array}{l}\text { gamma- } \\
\text { Cadinene }\end{array}$ & $\begin{array}{l}\text { Caryophyllene } \\
\text { oxide }\end{array}$ & $\begin{array}{l}\text { Humulene } \\
\text { epoxide II }\end{array}$ & $\begin{array}{c}\text { epi- } \\
\text { alpha- } \\
\text { Cadinol }\end{array}$ & $\begin{array}{l}\text { 14-hydroxy-Z- } \\
\text { Caryophyllene }\end{array}$ & $\begin{array}{l}\text { Hexadecanoic } \\
\text { acid }\end{array}$ & $\begin{array}{l}\text { Abieta- } \\
\text { 8(14),13(15- } \\
\text { diene) } \\
\end{array}$ & $\begin{array}{c}\text { Total } \\
\%\end{array}$ \\
\hline Manganese(Mn) & - & - & $0.23 b$ & - & $11.29 a$ & - & - & $0.67 a$ & - & $2.17 a b$ & 91.49 \\
\hline Boron (B) & $0.3 b$ & - & $0.22 b$ & - & $10.98 a$ & $0.56 b$ & - & $0.61 a$ & - & $2.17 a b$ & 90.25 \\
\hline Nitrogen $(\mathrm{N})$ & $1.5 a$ & $0.93 a$ & $0.3 a b$ & $1.46 a$ & $6.37 b$ & - & $0.47 b$ & $0.37 b$ & $0.27 b$ & - & 93.26 \\
\hline Phosphorus (P) & $0.71 b$ & $0.3 c$ & $0.4 a$ & $0.7 b$ & $5.62 b c$ & $0.64 a$ & $1.35 a$ & $0.64 a$ & $0.26 b$ & $1.57 b$ & 96.15 \\
\hline $\mathrm{Mn} \times \mathrm{B}$ & $1.2 a$ & $0.5 c$ & $0.3 a b$ & $1.12 a$ & $8.13 a b$ & $0.57 b$ & $0.27 b$ & $0.47 b$ & - & $1.22 b$ & 96.05 \\
\hline $\mathrm{Mn} \times \mathrm{N}$ & $1.3 b$ & $0.6 b$ & $0.23 b$ & $1.1 a$ & $3.1 c$ & $0.57 b$ & $0.2 b$ & $0.4 b$ & - & $1.27 b$ & 95.7 \\
\hline $\mathrm{Mn} \times \mathrm{P}$ & $0.8 b$ & $0.4 c$ & $0.28 b$ & - & $9.8 a b$ & $0.5 b$ & $0.27 b$ & $0.77 a$ & - & $1.4 b$ & 95.31 \\
\hline $\mathrm{B} \times \mathrm{N}$ & $0.5 b$ & - & $0.23 b$ & - & $11.4 a$ & $0.61 a b$ & - & $0.67 a$ & - & $2.4 a$ & 89.78 \\
\hline $\mathrm{B} \times \mathrm{P}$ & $1.5 a$ & - & $0.33 a b$ & - & $10.6 a$ & $0.22 c$ & $1.1 a$ & - & $0.3 a b$ & $1.37 b$ & 97.73 \\
\hline $\mathrm{N} \times \mathrm{P}$ & $1.5 a$ & - & $0.33 a b$ & - & $10.6 a$ & $0.22 c$ & $1.1 a$ & - & $0.37 b$ & $1.3 b$ & 84.11 \\
\hline $\mathrm{Mn} \times \mathrm{B} \times \mathrm{N}$ & $1.6 a$ & - & $0.41 a$ & - & $6.4 b$ & $0.77 a$ & $1.2 a$ & - & $0.3 a b$ & $1.6 b$ & 95.42 \\
\hline $\mathrm{Mn} \times \mathrm{B} \times \mathrm{P}$ & $1.45 a$ & - & $0.42 a$ & - & $8.3 a b$ & $0.47 b$ & $1.27 a$ & - & $0.31 a b$ & $2.37 a$ & 96.28 \\
\hline $\mathrm{Mn} \times \mathrm{N} \times \mathrm{P}$ & $1.5 \mathrm{a}$ & - & $0.43 a$ & - & $8.4 a b$ & $0.74 a$ & $1.1 a$ & - & $0.37 a$ & $2.6 a$ & 95.49 \\
\hline$B \times N \times P$ & $1.66 a$ & - & $0.45 a$ & - & $6.3 b$ & $0.71 a$ & $1.4 a$ & - & $0.43 a$ & $2.5 a$ & 93.22 \\
\hline $\mathrm{Mn} \times \mathrm{B} \times \mathrm{N} \times \mathrm{P}$ & $1.6 a$ & - & $0.43 a$ & - & $8.4 a b$ & $0.57 b$ & $1.76 a$ & - & $0.27 b$ & $2.67 a$ & 99.4 \\
\hline Control & $1.8 a$ & - & $0.44 a$ & - & $6.2 \mathrm{~b}$ & $0.6 a b$ & $1.4 a$ & - & $0.21 a$ & $1.5 b$ & 90.76 \\
\hline
\end{tabular}


Table 6a. Means of essential oil percentage measured in lemon balm plants that are affected by micronutrients (300 ppm) concentration and biofertilizeres ( $2^{\text {nd }}$ year).

\begin{tabular}{|c|c|c|c|c|c|c|c|c|c|c|c|}
\hline Treatments & $\begin{array}{l}\text { 6-methyl- } \\
\text { 5-Hepten- } \\
\text { 2-one }\end{array}$ & Linalool & $\begin{array}{c}\text { exo- } \\
\text { Isocitral }\end{array}$ & Citronellal & Z-Isoicitral & $\begin{array}{c}\text { Rosefuran } \\
\text { epoxide }\end{array}$ & E-Isoicitral & $\begin{array}{c}\text { Methyl } \\
\text { chavicol }\end{array}$ & Neral & Chavicol & Piperitone \\
\hline Manganese(Mn) & $1.4 c$ & $0.32 a b$ & $0.22 b$ & - & $1.5 b$ & $0.2 b$ & $1.7 a b$ & $0.75 c$ & $22.9 a b$ & - & $0.57 c$ \\
\hline Boron (B) & $0.4 d$ & $0.33 a b$ & $0.22 b$ & $0.32 c$ & $1.2 b$ & $0.22 b$ & $1.9 a b$ & $0.6 c$ & $19.5 b$ & $7.33 b$ & $0.64 c$ \\
\hline Nitrogen $(\mathrm{N})$ & $1.2 c$ & $0.39 a b$ & $0.22 b$ & $0.41 c$ & $1.2 b$ & $0.5 b$ & $1.5 b$ & $0.48 c$ & $14.4 b c$ & $12.28 a$ & $0.56 c$ \\
\hline Phosphorus (P) & $1.6 b c$ & $0.4 a b$ & - & $0.41 c$ & $1.1 b$ & $0.3 b$ & $1.6 a b$ & - & $27.8 a$ & - & $0.6 c$ \\
\hline $\mathrm{Mn} \times \mathrm{B}$ & $1.2 c$ & $0.4 a b$ & $0.28 b$ & $0.51 c$ & $1.59 b$ & $0.3 b$ & $2.3 a$ & $2.82 b$ & $14.5 b c$ & $12.65 a$ & $0.38 c$ \\
\hline $\mathrm{Mn} \times \mathrm{N}$ & $1.4 c$ & $0.56 a b$ & - & $0.4 c$ & $1.8 b$ & $0.3 b$ & $1.5 b$ & $2.1 b$ & $17.4 b$ & $6.16 b$ & $0.47 c$ \\
\hline $\mathrm{Mn} \times \mathrm{P}$ & $1.9 b$ & $0.39 a b$ & $0.21 b$ & $0.35 c$ & $1.1 b$ & $0.4 b$ & $1.5 b$ & $4.21 a$ & $26.5 a$ & - & $0.7 c$ \\
\hline $\mathrm{B} \times \mathrm{N}$ & $1.7 b c$ & $0.25 b$ & $0.22 b$ & $0.5 c$ & $1.28 b$ & $0.45 b$ & $1.9 a b$ & $4.31 a$ & $23.61 a b$ & - & $0.39 c$ \\
\hline $\mathrm{B} \times \mathrm{P}$ & $1.2 c$ & $0.48 a b$ & $0.23 b$ & $0.45 c$ & $1.18 b$ & $0.46 b$ & $1.6 a b$ & $0.15 c$ & $25.5 a b$ & - & $0.47 c$ \\
\hline $\mathrm{N} \times \mathrm{P}$ & $1.9 b$ & $0.79 a$ & - & $3.4 a$ & $5.1 a$ & $1.9 a$ & $1.45 b$ & - & $1.42 e$ & - & $2.3 a$ \\
\hline $\mathrm{Mn} \times \mathrm{B} \times \mathrm{N}$ & $1.6 b c$ & $0.49 a b$ & $0.22 b$ & $0.4 c$ & $1.27 b$ & $0.5 b$ & $1.8 a b$ & $0.58 c$ & $10.14 c$ & $12.28 a$ & $0.56 c$ \\
\hline $\mathrm{Mn} \times \mathrm{B} \times \mathrm{P}$ & $1.36 c$ & $0.49 a b$ & $0.21 b$ & $0.41 c$ & $1.2 b$ & $0.15 b$ & $1.6 a b$ & $0.5 c$ & $14.1 b c$ & $12.8 a$ & $0.5 c$ \\
\hline $\mathrm{Mn} \times \mathrm{N} \times \mathrm{P}$ & $2.6 a$ & $0.48 a b$ & - & $4.1 a$ & $3.1 a b$ & $1.3 a b$ & $1.65 a b$ & - & $22.41 a b$ & - & $1.4 b$ \\
\hline $\mathrm{B} \times \mathrm{N} \times \mathrm{P}$ & $1.9 b$ & $0.5 a b$ & - & $3.4 a$ & $5.1 a$ & $1.9 a$ & $1.45 b$ & $0.22 c$ & $12.42 b c$ & - & $1.3 b$ \\
\hline $\mathrm{Mn} \times \mathrm{B} \times \mathrm{N} \times \mathrm{P}$ & $1.5 b$ & $0.59 a b$ & $0.52 a$ & $3.34 a$ & $3.1 a b$ & $1.9 a$ & $1.9 a b$ & $0.62 c$ & $8.44 d$ & $10.1 a b$ & $0.56 c$ \\
\hline Control & $1.36 c$ & $0.49 a b$ & - & $2.9 a$ & $2.9 b$ & $1.6 a b$ & $1.8 a b$ & $0.28 c$ & $8.14 d$ & - & $0.56 c$ \\
\hline
\end{tabular}

Table 6b. Means of essential oil percentage measured in lemon balm plants that are affected by micronutrients (300 ppm) concentration and biofertilizeres ( $2^{\text {nd }}$ year).

\begin{tabular}{|c|c|c|c|c|c|c|c|}
\hline Treatments & Nerylformate & $\begin{array}{l}\text { Methyl } \\
\text { geranate }\end{array}$ & $\begin{array}{c}\text { Piperitenone } \\
\text { oxide }\end{array}$ & $\begin{array}{l}\text { Geranyl } \\
\text { acetate }\end{array}$ & E-Caryophyllene & $\begin{array}{l}\text { alpha-trans- } \\
\text { Bergamotene }\end{array}$ & $\begin{array}{c}\text { alpha- } \\
\text { Humulene }\end{array}$ \\
\hline Manganese(Mn) & $1.6 b$ & $0.4 a b$ & $2.2 a$ & $3.7 b$ & $7.2 a$ & $1.3 b$ & $1.52 a$ \\
\hline Boron (B) & $1.2 b$ & $0.3 b$ & $2.4 a$ & $3.4 b$ & $7.1 a$ & $1.3 b$ & $1.52 a$ \\
\hline Nitrogen $(\mathrm{N})$ & $2.1 a b$ & $0.5 a b$ & $2.2 a$ & $4.51 a b$ & $4.1 b$ & $1.3 b$ & $1.64 a$ \\
\hline Phosphorus (P) & $1.2 b$ & $0.3 b$ & $2.2 a$ & $3.4 b$ & $5.1 a b$ & $1.9 a$ & $1.45 a$ \\
\hline $\mathrm{Mn} \times \mathrm{B}$ & $2.2 a b$ & $0.5 a b$ & - & $7.3 a$ & $5.7 a b$ & $1.8 a$ & $1.5 \mathrm{a}$ \\
\hline $\mathrm{Mn} \times \mathrm{N}$ & $2.2 a b$ & $0.3 b$ & - & $5.9 a b$ & $6.3 a$ & $1.8 a$ & $1.66 a$ \\
\hline $\mathrm{Mn} \times \mathrm{P}$ & $2.2 a b$ & $0.3 b$ & - & $3.15 b$ & $6.9 a$ & $2.13 a$ & $1.6 a$ \\
\hline $\mathrm{B} \times \mathrm{N}$ & $1.6 b$ & $0.2 b$ & - & $3.65 b$ & $6.51 a$ & $1.9 a$ & $1.8 a$ \\
\hline $\mathrm{B} \times \mathrm{P}$ & - & - & $0.55 b$ & $6.7 a$ & $5.35 a b$ & - & $0.55 b$ \\
\hline $\mathrm{N} \times \mathrm{P}$ & $2.6 a b$ & $0.42 a b$ & - & $3.54 b$ & $5.6 a b$ & $0.3 c$ & $1.5 a$ \\
\hline $\mathrm{Mn} \times \mathrm{B} \times \mathrm{N}$ & $3.2 a$ & $0.42 a b$ & - & $3.43 b$ & $5.16 a b$ & $0.39 c$ & $1.5 a$ \\
\hline $\mathrm{Mn} \times \mathrm{B} \times \mathrm{P}$ & $2.1 a b$ & $0.4 a b$ & - & $4.1 a b$ & $5.1 a b$ & $1.33 b$ & $1.6 a$ \\
\hline $\mathrm{Mn} \times \mathrm{N} \times \mathrm{P}$ & $1.2 b$ & $0.39 a b$ & - & $3.43 b$ & $5.1 a b$ & $1.93 a$ & $1.45 a$ \\
\hline $\mathrm{B} \times \mathrm{N} \times \mathrm{P}$ & $2.2 a b$ & $0.53 a b$ & $2.3 a$ & $4.34 a b$ & $5.7 a b$ & $1.8 a$ & $1.5 \mathrm{a}$ \\
\hline $\mathrm{Mn} \times \mathrm{B} \times \mathrm{N} \times \mathrm{P}$ & $2.2 a b$ & $0.63 a$ & $1.7 a$ & $4.93 a b$ & $5.3 a b$ & $1.8 a$ & $1.26 a$ \\
\hline Control & $2.2 a b$ & $0.63 a$ & $1.6 a b$ & $4.1 b$ & $5.4 a b$ & $1.63 b$ & $1.1 a$ \\
\hline
\end{tabular}

Table 6c. Means of essential oil percentage measured in lemon balm plants that are affected by micronutrients $(300 \mathrm{ppm})$ concentration and biofertilizeres $\left(2^{\text {nd }}\right.$ year$)$.

\begin{tabular}{|c|c|c|c|c|c|c|c|c|}
\hline Treatments & $\begin{array}{l}\text { gamma- } \\
\text { Cadinene }\end{array}$ & Caryophyllene oxide & $\begin{array}{l}\text { Humulene } \\
\text { epoxide II }\end{array}$ & $\begin{array}{c}\text { epi-alpha- } \\
\text { Cadinol }\end{array}$ & $\begin{array}{l}\text { 14-hydroxy-Z- } \\
\text { Caryophyllene }\end{array}$ & Hexadecanoic acid & $\begin{array}{c}\text { Abieta- } \\
8(14), 13(15 \text {-diene })\end{array}$ & $\begin{array}{c}\text { Total } \\
\%\end{array}$ \\
\hline Manganese(Mn) & $1.2 a$ & $10.6 a$ & $0.12 c$ & $1.12 a$ & - & $0.23 a$ & $1.3 b$ & 89.69 \\
\hline Boron (B) & - & $10.6 a$ & $0.2 c$ & $1.21 a$ & - & $0.37 a$ & $1.32 b$ & 91.37 \\
\hline Nitrogen $(\mathrm{N})$ & - & $9.4 a$ & $0.37 b$ & $1.22 a$ & - & $0.32 a$ & $1.26 b$ & 95.49 \\
\hline Phosphorus (P) & $0.2 b$ & $8.1 a$ & $0.27 c$ & $1.27 a$ & - & $0.31 a$ & $1.37 b$ & 95.18 \\
\hline $\mathrm{Mn} \times \mathrm{B}$ & - & $3.4 c$ & $0.34 b$ & $1.12 a$ & - & $0.37 a$ & $1.6 a b$ & 96.49 \\
\hline $\mathrm{Mn} \times \mathrm{N}$ & - & $6.3 b$ & $0.51 a$ & $1.4 a$ & - & $0.43 a$ & $1.5 a b$ & 88.89 \\
\hline $\mathrm{Mn} \times \mathrm{P}$ & - & $8.4 a$ & $0.57 a$ & $1.76 a$ & - & $0.27 a$ & $2.6 a$ & 99.82 \\
\hline $\mathrm{B} \times \mathrm{N}$ & $1.2 a$ & $9.2 a$ & $0.6 a$ & $1.42 a$ & - & $0.41 a$ & $2.5 a$ & 90.52 \\
\hline $\mathrm{B} \times \mathrm{P}$ & - & $9.48 a$ & $0.51 a$ & - & $0.64 a$ & - & $2.4 a$ & 89.93 \\
\hline $\mathrm{N} \times \mathrm{P}$ & - & $9.6 a$ & $0.32 b$ & $1.1 a$ & - & $0.3 a$ & $1.3 b$ & 89.18 \\
\hline $\mathrm{Mn} \times \mathrm{B} \times \mathrm{N}$ & - & $8.6 a$ & $0.2 c$ & $1.12 a$ & - & $0.37 a$ & $1.3 b$ & 97.66 \\
\hline $\mathrm{Mn} \times \mathrm{B} \times \mathrm{P}$ & $1.1 a$ & $3.4 c$ & $0.47 a b$ & $1.2 a$ & - & $0.3 a$ & $1.6 a b$ & 95.95 \\
\hline $\mathrm{Mn} \times \mathrm{N} \times \mathrm{P}$ & $1.2 a$ & $6.3 b$ & $0.4 a b$ & $1.2 a$ & $0.2 b$ & $0.31 a$ & $2.3 a$ & 98.68 \\
\hline $\mathrm{B} \times \mathrm{N} \times \mathrm{P}$ & $1.1 a$ & $6.1 b$ & $0.44 a b$ & - & $0.5 b$ & $0.3 a$ & $2.2 a$ & 96.39 \\
\hline $\mathrm{Mn} \times \mathrm{B} \times \mathrm{N} \times \mathrm{P}$ & $1.2 a$ & $6.3 b$ & $0.41 a b$ & $1.14 a$ & $0.7 a$ & $0.4 a$ & $1.25 a b$ & 98.99 \\
\hline Control & $1.1 a$ & $6.4 b$ & $0.17 c$ & $1.06 a$ & - & $0.27 a$ & $1.6 a b$ & 88.72 \\
\hline
\end{tabular}

Numbers in each column that have same word, have same group. ns, *and** : Significant at $\mathrm{P}=0.05$ and $\mathrm{P}=0.01$ levels of probability, respectively. 


\section{Discussion}

In the present study, the effect of micronutrients applications with macronutrients (biofertilizeres) was determined on leaf dry/fresh weight, root dry/fresh weight, stem/root length, number of stem and essential oils of Lemon balm. The most important characters in this research was on essence components. In the soil application, the nutrients can be bound and therefore cannot be available for the plants. Also, since most nutrients are taken up with water, their uptake is restricted during water stress. The critical levels micronutrients were not determined for Lemon balm plants. In other hand, it was deduced dual inoculation with beneficial bacteria (Bacillus sp. N1-19NA, Enterobacter sp. N0-29PA, Pseudomonas sp.N155PA and Serratia sp. N0-10LB) are able to compensate the lack nutrient in soils (Martinez et al. 2015). Yield Enhancement in field trials was produced by using a combination of Bacillus licheniformis, in powder form and Azospirillum and Azotobacter. Strains of bacteria have increased root and shoot elongation. The biofertilizer therefore may have a potential to decrease the input cost of agricultural production, and be applied to the re-vegetation of low commercial value sites, such as metal tailings ponds (Yadegari et al. 2010). The effect of biofertilizeres on growth properties, yield and quality property in medicinal plants was studied by other researchers and they showed same beneficial effects of biofertilizeres (Viruel et al. 2014). Patora et al. (2003) on Lemon balm and Yadegari (2015) on borage, thyme and marigold showed beneficial application from micronutrients. The best treatment which was achieved maximum essence components of shoots was using biofertilizeres with micronutrients simultaneously. However, the L.S.D multiple range results revealed using biofertilizeres with micronutrients had the most influence on essence components of shoots and in various treatments, the treat had $150 \mathrm{ppm}$ of $\mathrm{Mn}$ and $150 \mathrm{ppm}$ of B with use of biofertilizeres was the best (Tables 3-6). The most biological yield was obtained by biofertilizeres (Yadegari et al. 2010). The enhanced phosphatase activity may help the plant to mobilize P and thereby increase the biomass production (Viruel et al. 2014). The significant effect that $\mathrm{H}_{2} \mathrm{Bo}^{3-}$ and $\mathrm{Mn}^{2+}$ by biofertilizeres consumption had on essence and growth parameters such as leaf dry/fresh weight, root dry/fresh weight, stem/root length, number of stem, indicates that micro/macronutrients play an important role in the yield of Lemon balm. Lemon balm needs $\mathrm{No}^{3-}$, $\mathrm{Po}_{4}{ }^{3-}, \mathrm{H}_{2} \mathrm{Bo}^{3-}$ and $\mathrm{Mn}^{2+}$ and it is possible that this requirement increases during growth, as the beginning of flowering process and the reproductive tissue have higher requirements for $\mathrm{H}_{2} \mathrm{Bo}^{3-}$ and $\mathrm{Mn}^{2+}$ foliar applications can have a direct effect on yield. In this study the response to micro/macronutrient sapplications can be higher. This is possible since the plants grew better with the macronutrients from biofertilizeres resource and micronutrients $\left(\mathrm{H}_{2} \mathrm{Bo}^{3-}\right.$ and $\left.\mathrm{Mn}^{2+}\right)$ application, developed a bigger root system, and took up more nutrients. $\mathrm{H}_{2} \mathrm{Bo}^{3-}$ and $\mathrm{Mn}^{2+}$ are immobile in plants and can't be transported to developing organs. Therefore, foliar application can provide $\mathrm{H}_{2} \mathrm{Bo}^{3-}$ and $\mathrm{Mn}^{2+}$ to the developing organs that need it the most and, in this case, the plants do not have taken these micronutrients from the soil solution. However, the actual amount of $\mathrm{H}_{2} \mathrm{Bo}^{3-}$ and $\mathrm{Mn}^{2+}$ that reaches the reproductive tissue can be small and depends on other factors such as $\mathrm{H}_{2} \mathrm{Bo}^{3-}$ and $\mathrm{Mn}^{2+}$ soil and plant levels, and water stress that can restrict micronutrients movement and this is possibly one reason for finding a significant increase in yield because of the micronutrients application. Micro/macronutrients application affected exo-Isocitral, Chavicol, 14-hydroxy-Z-Caryophyllene and Germacrene D as the plants in the control treatment and increased with biofertilizeres with micronutrients applications and no detected in control plants (Tables 3-6). 
In same research, the effect of farm yard manure reported significant on neral, $(28.43 \%)$, geranial $(39.86 \%)$ and geranyl acetate $(8.67 \%)$ compared to other treatments (Harshavardhan et al. 2007).

When the nitrogen, phosphorus, boron and manganese level is too low to sustain plant growth, the plants become shorter and the total biomass is lower compared with the plants with sufficient micronutrients (Mengel et al. 2001; Habib, 2012). This effect can be explained as micro/macronutrients affecting the carbohydrate transport, and this can affect the yield components in many plants. Since micro/macronutrients affects the meristems, the increase in the availability of micro/ macronutrients can increase the number of stems per plant as they grow better.Galavi et al. (2012) on safflower, Yarnia et al. (2012) on Purple coneflower, Younis et al. (2013) on Rosa hybrida, Marquez et al. (2015) on cowpea, Yadegari (2015) on borage, thyme and marigold, found that spraying micronutrients increased essential oils in plants. The essential oil yield increased with $\mathrm{H}_{2} \mathrm{Bo}^{3-}$ and $\mathrm{Mn}^{2+}$ applications with macronutrients, because there was a significant increase in dry matter yield. However, more research is needed to explore these tools for Lemon balm breeding and for better Lemon balm management. There are still many unanswered questions about how biofertilizeres with $\mathrm{H}_{2} \mathrm{Bo}^{3-}$ and $\mathrm{Mn}^{2+}$ act in increasing essential oils for Lemon balm. One possibility is that the applied micronutrients can affect dry matter accumulation and by macronutrients consumption increase dry matter yield. Macro/micronutrients can increase the number of stems per plant and slowdown leaf senescence (Yadegari, 2015). The obtained results are in conformity with those of Sari and Ceylan (2002) on lemon balm and Yadegari (2015) on borage, thyme and marigold. The main components of the oil were Caryophyllene oxide, E-Caryophyllene, Geranial, Geraniol, Chavicol and Neral for all treatments. Many researchers have reported that the main components of lemon balm are
Caryophyllene oxide, E-Caryophyllene, Geranial, Geraniol, Chavicol and Neral (Manukyan, 2011; Patora et al. 2003; Sari and Ceylan, 2002). However, there were significant differences among the rates of those reported components. Neral and geranial rates in the oil were reported respectively as $15 \%$ and $14.5 \%$ by Hefendehl (1970), 19.6-36.1\% and 25.3-47.5\% by Tittel et al. (1982), 19.5\% and $31.6 \%$ by Werker et al. (1985), $7.195 \%$ and $30-40 \%$ and $50-60 \%$ by Ceylan et al. (1994). Although many studies discussed above reported that neral and geranial were the main components of the oil, Kirimer et al. (1995) found that the main component of the lemon balm oil they studied in some of studies citronellal, linalool and geranial as major chemical compositions of the essential oil of the lemon balm (Adinee et al. 2008; Bagdat and Cosge, 2006; Mrlianova et al. 2001; Sorensen, 2000). All the significant differences for the components of the oil among the papers discussed above may be due to the use of different genetic material and/or different environmental conditions.

\section{Conclusions}

This study showed that the applications macronutrients $\left(\mathrm{PO}_{4}{ }^{3-}\right.$ and $\left.\mathrm{NO}^{3-}\right)$ from biofertilizeres with micronutrients $\left(\mathrm{H}_{2} \mathrm{Bo}^{3-}\right.$ and $\left.\mathrm{Mn}^{2+}\right)$ had significant effects on growth parameters such as leaf/root dry/fresh weight, stem/root length, number of stem and essential oils of Lemon balm such as trans-Piperitone epoxide percentage. The main components of the oil were Caryophyllene oxide, E-Caryophyllene, Geranial, Geraniol, Chavicol and Neral for all treatments. This indicates that application of micronutrients and soil consumption of macronutrients from biofertilizeres have significant effects on the composition of the essential oil. Results obtained from this study showed that the essential oils composition of lemon balm were significantly affected by application of micronutrients and 
biofertilizeres. The highest amount of essential oil compositions such as Linalool, Z-Isoicitral, Rosefuran epoxide, E-Isoicitral, alpha-trans-Bergamotene, alpha-Humulene, epi-alpha-Cadinol, Hexadecanoic acid and Abieta-8 (14),13 (15-diene) obtained from combination of treatments of micronutrients and biofertilizeres application in two cultivation seasons. This study provides some useful information about the effects of application of $\mathrm{PO}_{4}^{3-}, \mathrm{NO}^{3-}, \mathrm{H}_{2} \mathrm{Bo}^{3-}$ and $\mathrm{Mn}^{2+}$ on Lemon balm production and in that way increases our knowledge about the effect of micro/macronutrients on crop production.

\section{Acknowledgement}

This work was supported by the Islamic Azad University Branch of Shahrekord, Iran. We are very grateful to Mrs. P. Karimi from Education Center of Shahrekord for helpful advice for secondary reviewer.

\section{References}

Adams, R.P. 2001. Identification of Essential Oil Components by Gas Chromatography/ Mass Spectroscopy. Allured publishing Corp. Carol Stream. USA.

Adak, T., Singha, A., Kumar, K., Shukla, S.K., Singh, A., Kumar Singh, V. 2014. Soil organic carbon, dehydrogenase activity, nutrient availability and leaf nutrient content as affected by organic and inorganic source of nutrient in mango orchard soil. Journal of Soil Science and Plant Nutrition. 14(2): 394-406.

Adinee, J., Piri, K.H.,Karami, O. 2008. Essential Oil Component in Flower of Lemon Balm (Melissa officinalis L.). American Journal of Biochemistry and Biotechnology. 4(3): 277-278.
Bagdat, R.B.,Cosge, B. 2006. The essential oil of Lemon balm (Melissa officinalis L.), its components and using fields. Journal of Faculty of Agriculture OMU. 21(1): 116-121.

Ceylan, A., Bayram, E.,Ozay, N. 1994. Melissa offcinalis L. (Oguloto) in agronomikveTeknolojikOzellikleri Uzerinde Arastirmalar. Doga, Turkish Journal of Agricultural and Forestry. 18, 125-130.

Galavi, M., Ramroudi, M.,Tavassoli, A. 2012. Effect of micronutrients foliar application on yield and seed oil content of safflower (Carthamus tinctorius). African Journal of Agricultural Research. 7(3): 482-486.

Ge, P., Da, L.G., Wang, W.B., Xu, X.N. 2014. Seasonal dynamics of dissolved organic carbon, nitrogen and other nutrients in soil of Pinus massoniana stands after pine wilt disease disturbance. Journal of Soil Science and Plant Nutrition. 14(1): 75-87.

Habib, M. 2012. Effect of supplementary nutrition with $\mathrm{Fe}, \mathrm{Zn}$ chelates and urea on wheat quality and quantity. African Journal of Biotechnology. 11 (11): 2661-2665.

Harshavardhan, P.G., Vasundhara, M., Sreenivasappa, K.N. 2007. Influence of spacing and integrated nutrient management on yield and quality of essential oil in lemon balm (Melissa officinalis L). BIOMED. 2(3): 288-293.

Hefendehl, F.W. 1970. Zusammensetzung des atherischen Ols von Melissa officinalis L. und sekundareVeranderungen der Olkomposition. Arch. Pharm. 303, 345-357.

Khan, I., Zaman, M., Khan, M.J., Iqbal, M., Babar, M.N. 2014. How to improve yield and quality of potatoes: effects of two rates of urea N, urease inhibitor and Cytozyme nutritional program. Journal of Soil Science and Plant Nutrition. 14 (2): 268-276. http://dx.doi.org/10.4067/S071895162014005000022 . 
Kirimer, N., Baser, K.H.C.,Tumen, G. 1995. Carvacrolrich plants in Turkey. Khim. Prir. Soedin. 4: 49-54.

Manukyan, A. 2011. Effect of growing factors on productivity and quality of Lemon catmint, Lemon balm and Sage under soilless greenhouse production: I. Drought stress. Medicinal and Aromatic Plant Science and Biotechnology. 5(2): 119-125.

Martínez, O.A., Crowley, D.E., Mora, M.L., Jorquera, M.A. 2015. Short-term study shows that phytatemineralizing rhizobacteria inoculation affects the biomass, phosphorus (P) uptake and rhizosphere properties of cereal plants. Journal of Soil Science and Plant Nutrition. 15(1): 153-166.

Márquez-Quiroz, C., De-la-Cruz-Lázaro, E., OsorioOsorio, R., Sánchez-Chávez, E. 2015. Biofortification of cowpea beans with iron: iron's influenceon mineral content and yield. Journal of Soil Science and Plant Nutrition. 15 (4): 839-847.

Mengel, K., Kirkby, E.A., Kosegarten, H.,Appel, T. 2001. Principles of Plant Nutrition. Kluwer Academic Publishers, London.

Mrlianova, M., Tekelova, D.,Grancai, D. 2001. Comparison of the quality of Melissa officinalis L. cultivar Citra with Mellissas of European origin. Pharmacospsychiatry. 34: 20-21.

Patora, J., Majda, T., Gora, J.,Klimek, B. 2003. Variability in the content and composition of essential oil from Lemon balm (Melissa officinalis L.) cultivated in Poland. Acta Poloniae Pharmaccutica. 60(5): 395-400.

Quezada, C., Vidal, I., Lemus, L., Sanchez, H. 2007. Effect of nitrogen fertilization on yield and fruit quality of raspberries (Rubusidaeus L.) under two fertigation programs. R.C. SueloNutr. Veg. 7(3): 1-15. ISSN 0718-2791. http://dx.doi.org/10.4067/ S0718-27912007000300001.

Raza, M.A.S., Saleem, M.F., Shah, G.M., Khan, I.H., Raza, A. 2014. Exogenous application of glycinebetaine and potassium for improving water relations and grain yield of wheat under drought. Journal of Soil Science and Plant Nutrition. 14(2): 348-364. http://dx.doi.org/10.4067/S0718 95162014005000028 .

Rengel, Z. 2015. Availability of Mn, Zn and Fe in the rhizosphere. Journal of Soil Science and Plant Nutrition. 15 (2): 397-409. Sandana, P., Pinochet, D. 2014. Grain yield and phosphorus use efficiency of wheat and pea in a high yielding environment. J. Soil Sci. Plant Nutr.14 (4): 973986. ISSN 0718-9516. http://dx.doi.org/10.4067/ S0718-95162014005000076.

Sari, A.O.,Ceylan, A. 2002. Yield Characteristics and Essential Oil Composition of Lemon balm (Melissa officinalis L.) Grown in the Aegean Region of Turkey. Turkish Journal of Agriculture. 26: 217-224.

Sekeroglu, N., Alpaslan, D., Kirpik, M. 2006. Essential oil contents and ethno pharmacological characteristics of some species and herbal drugs traded in turkey. International Journal of Pharmacology. 2(2): 256-261.

Sorensen, J.M. 2000. Melissa officinalis, Essential oil-authenticity, production and pharmacological activity. International Journal Aromatherapy. 10: $1-8$.

Sun, S., Xing, F., Zhao, H., Gao, Y., Bai1, Z., Dong, Y. 2014. Response of bacterial community to simulated nitrogen deposition in soils and a unique relationship between plant species and soil bacteria in the Songnen grassland in Northeastern China. Journal of Soil Science and Plant Nutrition. 14(3): 565-580.

Tittel, G., Wagner, H., Bos, R. 1982. Chemical composition of the essential oil from Melissa. Planta Medica. 46, 91-98.

Viruel, E., Erazzú, L.E., Martínez Calsina, L., Ferrero, M.A., Lucca, M.E.,Siñeriz, F.E. 2014. Inoculation of maize with phosphate solubilizing 
bacteria:effect on plant growth and yield. Journal of Soil Science and Plant Nutrition. 14 (4): 819831.

Yadegari, M., Asadi Rahmani, H., Noormohammadi, G. 2010. Plant growth promoting Rhizobacteria increase growth, yield and nitrogen fixation in Phaseolus vulgaris. Journal of Plant Nutrition. 33, 1733-1743.

Yadegari, M. 2015. Foliar application of micronutrients on essential oils of borago, thyme and marigold. Journal of Soil Science and Plant Nutrition. 15, 949-964.

Yañez-Mansilla, E., Cartes, P., Reyes-Díaz, M., Ribera-Fonseca, A., Rengel, Z., Alberdi, M. 2015. Leaf nitrogen thresholds ensuring high antioxidant features of Vaccinium corymbosum cultivars. J. Soil Sci. Plant Nutr. 15, 3, 574-586.

Yarnia, M., Farzanian, M., Aliasgharzad, N. 2012. Effects of microelement fertilizers and phosphate biological fertilizer on some morphological traits of Purple coneflower in water stress condition. African Journal of Microbiology Research. 6, 4825-4832.
Younis, A., Riaz, A., Sajid, S.,Nadeem, M. 2013. Foliar application of macro- and micronutrients on the yield and quality of Rosa hybridacvs. Cardinal and Whisky Mac. African Journal of Biotechnology. 12(7): 702-708.

Werker, E., Ravid, U., Putievsky, E. 1985. Structure of glandular hairs and identification of the main components of their secreted material in some species of the Labiatae. Israel Journal of Botany. $34,31-45$.

Widom, M., Mihalkovic, M. 2008. Symmetry-broken crystal structure of elemental boron at low temperature. Physiology Review B. 77 (6): 24-34.

Wyllyam Do Vale, D., De Mello Prado, R., Natale, W., Ursulino Alves, A. 2008. Nutritional response of rootstock of lemon 'citrumelo' to nitrogen, phosphorus and potassium application. R.C. Suelo Nutr. Veg. 8(3): 40-48. ISSN 0718-2791. http://dx.doi.org/10.4067/S071827912008000300004. 